\title{
Broad sarbecovirus neutralization by a human monoclonal antibody
}

https://doi.org/10.1038/s41586-021-03817-4

Received: 29 March 2021

Accepted: 9 July 2021

Published online: 19 July 2021

Check for updates

\author{
M. Alejandra Tortorici ${ }^{1,2,9}$, Nadine Czudnochowski ${ }^{3,9}$, Tyler N. Starr $^{4,9}$, Roberta Marzi ${ }^{5,9}$, \\ Alexandra C. Walls' ${ }^{1}$ Fabrizia Zatta ${ }^{5}$, John E. Bowen', Stefano Jaconi ${ }^{5}$, Julia Di lulio ${ }^{3}$, \\ Zhaoqian Wang', Anna De Marco ${ }^{5}$, Samantha K. Zepeda', Dora Pinto ${ }^{5}$, Zhuoming Liu', \\ Martina Beltramello ${ }^{5}$, Istvan Bartha ${ }^{5}$, Michael P. Housley ${ }^{3}$, Florian A. Lempp ${ }^{3}$, Laura E. Rosen ${ }^{3}$, \\ Exequiel Dellota $\mathrm{Jr}^{3}$, Hannah Kaiser ${ }^{3}$, Martin Montiel-Ruiz ${ }^{3}$, Jiayi Zhou ${ }^{3}$, Amin Addetia ${ }^{4}$, \\ Barbara Guarino ${ }^{3}$, Katja Culap ${ }^{5}$, Nicole Sprugasci ${ }^{5}$, Christian Saliba ${ }^{5}$, Eneida Vetti ${ }^{5}$, \\ Isabella Giacchetto-Sasselli ${ }^{5}$, Chiara Silacci Fregni ${ }^{5}$, Rana Abdelnabi ${ }^{7}$, Shi-Yan Caroline Foo ${ }^{7}$, \\ Colin Havenar-Daughton ${ }^{3}$, Michael A. Schmid ${ }^{5}$, Fabio Benigni ${ }^{5}$, Elisabetta Cameroni ${ }^{5}$, \\ Johan Neyts ${ }^{7}$, Amalio Telenti ${ }^{3}$, Herbert W. Virgin ${ }^{3}$, Sean P. J. Whelan ${ }^{6}$, Gyorgy Snell ${ }^{3}$,

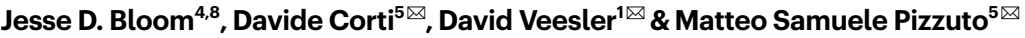

\begin{abstract}
The recent emergence of SARS-CoV-2 variants of concern ${ }^{1-10}$ and the recurrent spillovers of coronaviruses ${ }^{11,12}$ into the human population highlight the need for broadly neutralizing antibodies that are not affected by the ongoing antigenic drift and that can prevent or treat future zoonotic infections. Here we describe a human monoclonal antibody designated S2X259, which recognizes a highly conserved cryptic epitope of the receptor-binding domain and cross-reacts with spikes from all clades of sarbecovirus. S2X259 broadly neutralizes spike-mediated cell entry of SARS-CoV-2, including variants of concern (B.1.1.7, B.1.351, P.1, and B.1.427/B.1.429), as well as a wide spectrum of human and potentially zoonotic sarbecoviruses through inhibition of angiotensin-converting enzyme 2 (ACE2) binding to the receptor-binding domain. Furthermore, deep-mutational scanning and in vitro escape selection experiments demonstrate that $\mathbf{2} 2 \mathrm{X} 259$ possesses an escape profile that is limited to a single substitution, G504D. We show that prophylactic and therapeutic administration of S2X259 protects Syrian hamsters (Mesocricetus auratus) against challenge with the prototypic SARS-CoV-2 and the B.1.351 variant of concern, which suggests that this monoclonal antibody is a promising candidate for the prevention and treatment of emergent variants and zoonotic infections. Our data reveal a key antigenic site that is targeted by broadly neutralizing antibodies and will guide the design of vaccines that are effective against all sarbecoviruses.
\end{abstract}

SARS-CoV-2 has caused a global pandemic with over 182 million infections and more than 3 million fatalities as of July 2021. SARS-CoV-2 genetic drift has resulted in emerging variants of concern that are characterized by higher transmissibility, immune evasion and disease severity relative to the ancestral isolate ${ }^{1-10}$. Countermeasures, such as vaccines and therapeutic agents, are needed to cope with SARS-CoV-2 evolution and to protect against future sarbecovirus spillovers.

The sarbecovirus spike (S) glycoprotein mediates viral entry into host cells, represents the main target of neutralizing antibodies and is the focus of vaccine design ${ }^{11}$.S comprises an $\mathrm{S} 1$ subunit, which recognizes host cell receptors, and an S2 subunit that promotes viral-cell membrane fusion ${ }^{12,13}$. The S1 subunit includes the receptor-binding domain (RBD), which in the case of SARS-CoV and SARS-CoV-2 interacts with ACE2 to allow virus entry ${ }^{12,14-16}$.
A highly conserved region on sarbecovirus RBDs, designated antigenic site $\mathrm{II}^{17}$, has previously been shown to elicit SARS-CoV and SARS-CoV-2 cross-neutralizing antibodies ${ }^{18,19}$. However, site II becomes accessible only when at least two RBDs in the S trimer adopt an open conformation and thus has limited immunogenicity ${ }^{17,19}$. Here we describe a site-II-targeting monoclonal antibody $(\mathrm{mAb})$ designated S2X259 that possesses exceptional neutralization breadth within the Sarbecovirus subgenus (including all SARS-CoV-2 variants of concern), and an escape profile that is limited to a sole and rare substitution. In addition, we show that prophylactic and therapeutic administration of S2X259 protects Syrian hamsters against SARS-CoV-2 challenge with the prototypic virus and the B.1.351 variant of concern. Our findings identify S2X259 as a promising countermeasure to protect against SARS-CoV-2 antigenic drift as well as potentially new zoonotic

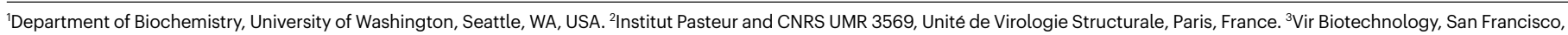
CA, USA. ${ }^{4}$ Basic Sciences Division and Computational Biology Program, Fred Hutchinson Cancer Research Center, Seattle, WA, USA. ${ }^{5} \mathrm{Humabs}$ Biomed SA, a subsidiary of Vir Biotechnology, Bellinzona, Switzerland. ${ }^{6}$ Department of Molecular Microbiology, Washington University School of Medicine, St Louis, MO, USA. ${ }^{7}$ Rega Institute for Medical Research, Laboratory of Virology and Chemotherapy, KU Leuven, Leuven, Belgium. ${ }^{8}$ Howard Hughes Medical Institute, Seattle, WA, USA. ${ }^{9}$ These authors contributed equally: M. Alejandra Tortorici, Nadine Czudnochowski, Tyler N. Starr, Roberta Marzi. ${ }^{凶}$-mail: dcorti@vir.bio; dveesler@uw.edu; mpizzuto@vir.bio 

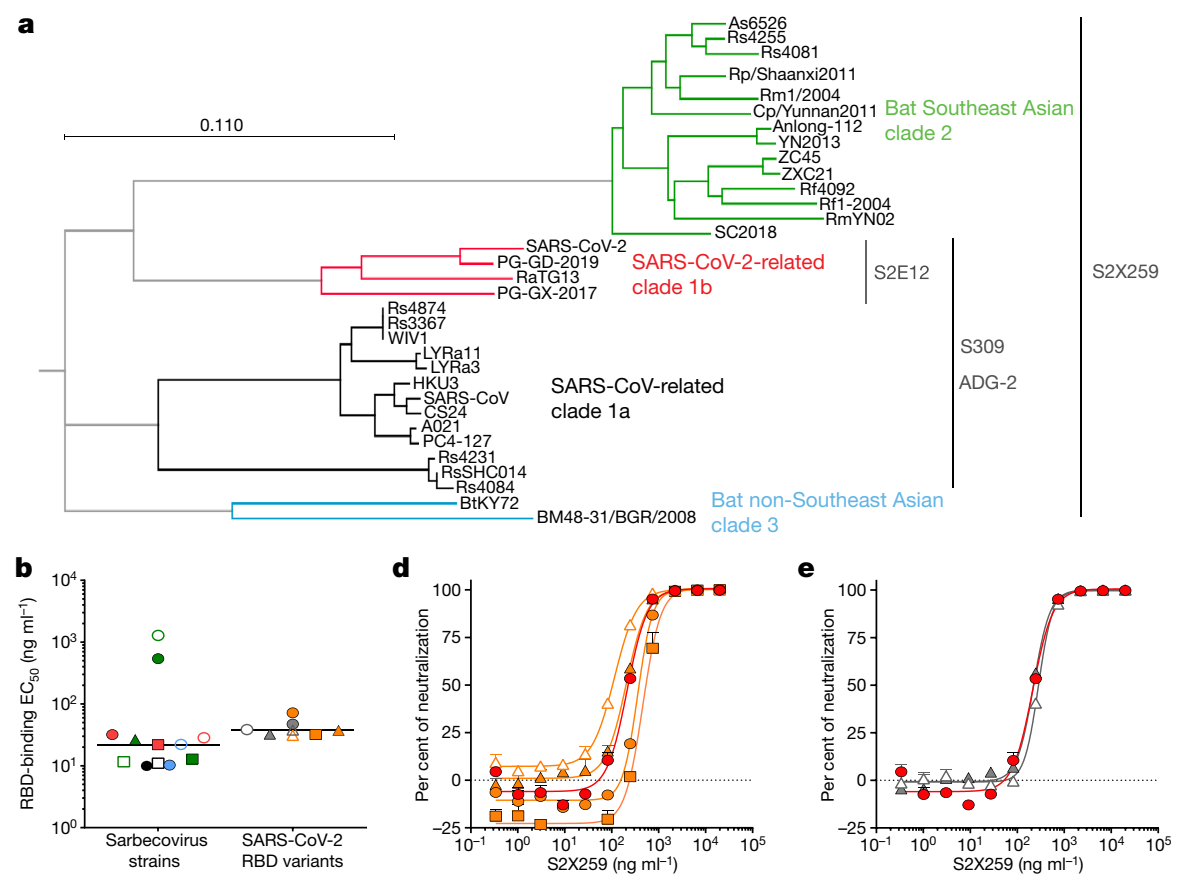

e

c
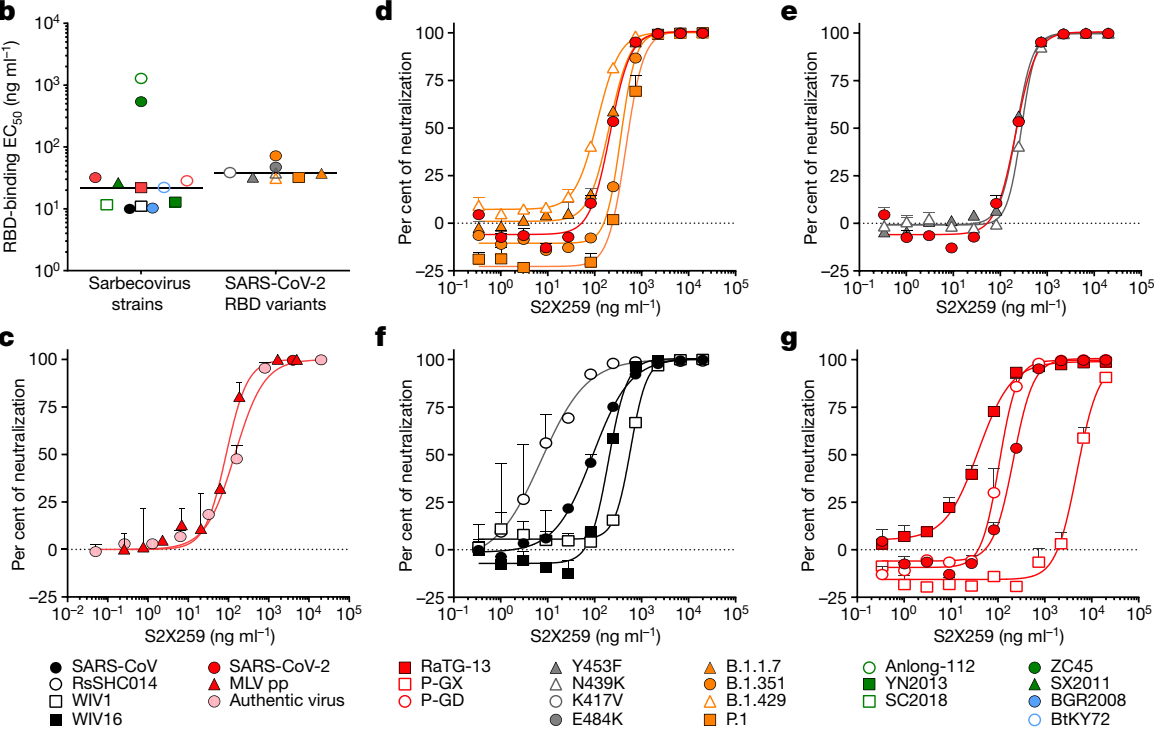

f

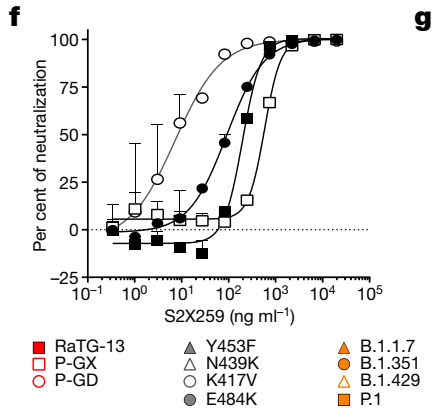

g

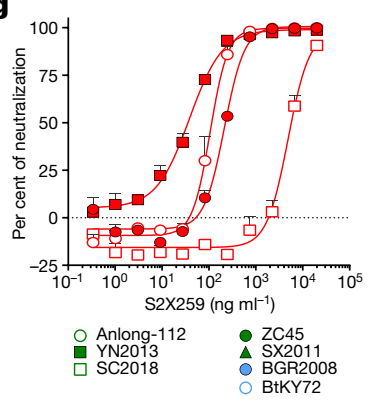

Fig. 1 | Identification of a broadly neutralizing sarbecovirus mAb. a, Phylogenetic tree of sarbecovirus RBDs constructed using maximum likelihood analysis of amino acid sequences retrieved from GISAID and GenBank. Cross-reactivity within the Sarbecovirus subgenus for $\mathrm{S} 2 \mathrm{E} 12^{21}, \mathrm{~S}_{30} 9^{22}$ and ADG-20 is included for comparison. b, S2X259 binding to RBDs representative of the different sarbecovirus clades and SARS-CoV-2 variants as measured by ELISA.c, S2X259-mediated neutralization of SARS-CoV-2-Nluc authentic virus and SARS-CoV-2 S MLV-pseudotyped virus (MLV pp). d, e, S2X259-mediated neutralization of VSV pseudotypes containing SARS-CoV-2 S from isolates representing the B.1.1.7, B.1.351, P.1 and B.1.429 variants of concern (d) as well as single RBD mutants (e).f, g, S2X259-mediated neutralization of VSV pseudotypes containing SARS-CoV-related (clade 1a) (f) or SARS-CoV-2-related (clade 1b) (g) S glycoproteins. $n=2$ independent experiments. Error bars indicate s.d. of duplicates or triplicates. infections, and highlight the importance of RBD site II for the design of vaccines eliciting antibodies against all sarbecoviruses.

\section{A broadly neutralizing sarbecovirus $\mathrm{mAb}$}

We identified the mAb designated S2X259 from the memory B cells of an individual who had recovered from COVID-19 (Supplementary Table 1); this mAb cross-reacted with 25 out of $26 \mathrm{~S}$ glycoproteins representative of all clades of sarbecovirus ${ }^{18,20}$ (Fig. 1a, Extended Data Fig. 1a-c). However, no binding was observed to embecovirus S (HCoV-HKU1 and HCoV-OC43) or merbecovirus S (MERS-CoV) glycoproteins (Extended Data Fig. 1a, b).

The cross-reactivity of S2X259 within the Sarbecovirus subgenus was further confirmed using enzyme-linked immunosorbent assay (ELISA) with a panel of 12 RBDs (Fig. 1b). S2X259 bound tightly to all of the RBDs tested (half maximal effective concentration $\left(\mathrm{EC}_{50}\right.$ ) of less than $32 \mathrm{ng} \mathrm{ml}^{-1}$ ) with the exception of two bat strains, ZC45 (EC ${ }_{50}$ of $539 \mathrm{ng}$ $\mathrm{ml}^{-1}$ ) and Anlong-112 (EC $\mathrm{E}_{50}$ of 1,281 $\mathrm{ng} \mathrm{ml}^{-1}$ ) (Fig. 1b). The broad recognition of S from the divergent bat Southeast Asian and non-Southeast Asian clades (clades 2 and 3, respectively) highlights the exceptional breadth of S2X259 in comparison with previously reported $\mathrm{mAbs}^{17,21,22}$
(Extended Data Fig. 1d). Surface plasmon resonance indicated that the S2X259 Fab fragment bound to clade $1 \mathrm{a}, 1 \mathrm{~b}$ and 3 RBDs with nano- to picomolar affinities, and to clade 2 RBDs with micro- to nanomolar affinities. Of note, the affinity of S2X259 for the RBD of the Guanxi pangolin coronavirus was 10-100-fold lower compared to other clade $1 b$ RBDs (Extended Data Fig. 2a). The S2X259 Fab recognized both the SARS-CoV-2 prefusion-stabilized Sectodomain trimer and the recombinant RBD with picomolar affinities, albeit with a slower on-rate for binding to $S$ (presumably owing to limited epitope exposure) ${ }^{12,13}$ (Extended Data Fig. 2a). Finally, S2X259 binding was unaffected by several point mutants or constellation of mutations identified in circulating clinical isolates (K417V, N439K, Y453F and E484K) and in the RBDs of the B.1.1.7 (N501Y), B.1.351 (K417N/E484K/N501Y), B.1.427/B.1.429(L452R) and P.1 (K417T/E484K/N501Y) lineages (Fig. 1b, Extended Data Fig. 2b).

We next evaluated the neutralization potency of S2X259 using authentic SARS-CoV-2-Nluc as well as murine leukaemia virus (MLV) and vesicular stomatitis virus (VSV) pseudotyping systems. S2X259 potently neutralized authentic SARS-CoV-2, SARS-CoV-2 S MLV and SARS-CoV-2 S VSV with half-maximal inhibitory concentrations $\left(\mathrm{IC}_{50}\right)$ of $144 \mathrm{ng} \mathrm{ml}^{-1}, 87 \mathrm{ng} \mathrm{ml}^{-1}$ and $213 \mathrm{ng} \mathrm{ml}^{-1}$, respectively (Fig. 1c, d). We subsequently evaluated S2X259-mediated neutralization against VSV 

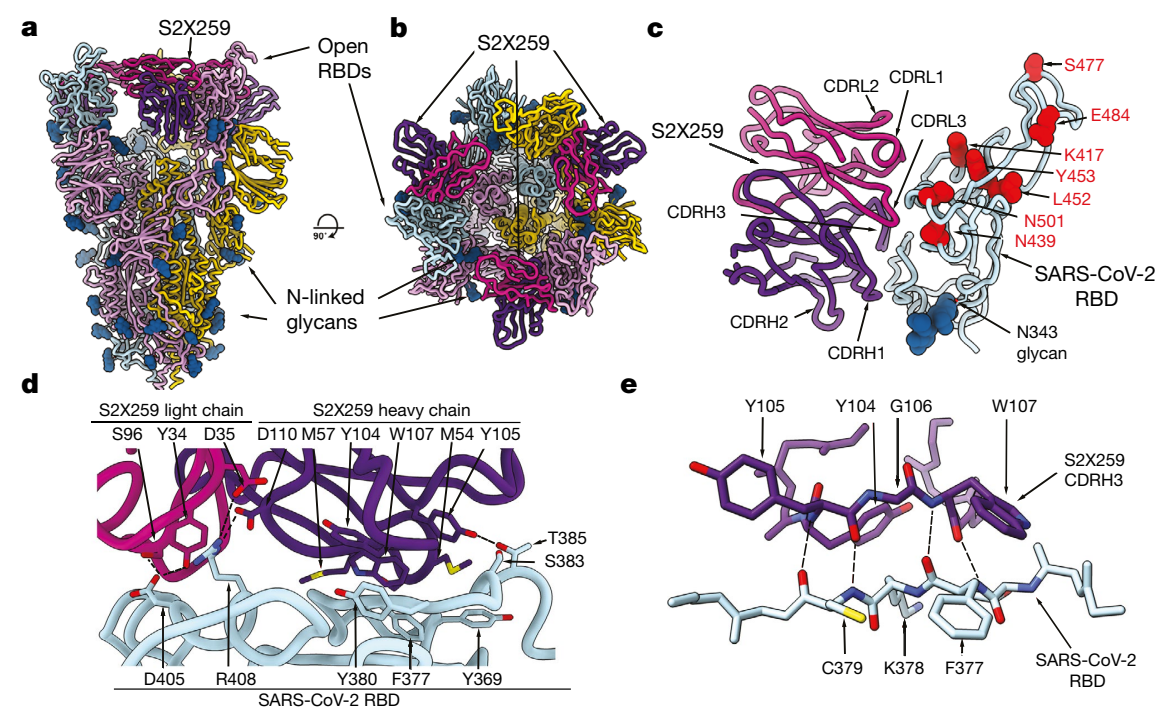

Fig. 2 | The S2X 259 broadly neutralizing sarbecovirus $m A b$ recognizes RBD antigenic site II. a, b, Cryo-electron microscopy structure of the prefusion SARS-CoV-2S ectodomain trimer with three S2X259 Fab fragments bound to three open RBDs, viewed along two orthogonal orientations (Protein Data Bank (PDB) codes 7RA8 and 7RAL).c, The S2X259 binding mode involving contacts with multiple RBD regions. Residues corresponding to prevalent RBD

pseudotypes containing SARS-CoV-2 S from the B.1.1.7, B1.351, P.1, B.1.427/B.1.429 and B.1.617.1 and other lineages, as well as the $\mathrm{N} 439 \mathrm{~K}$ and Y453F single mutations ${ }^{5,23-25}$. S2X259 neutralized all of these mutants with a similar potency, consistent with the identical binding affinities determined using RBD mutants (Fig. 1d, e, Extended Data Figs. 2b, 3a, b, Supplementary Table 2). Furthermore, S2X259 also neutralized a broad panel of ACE2-using sarbecoviruses with comparable potencies, including Guangdong pangolin coronavirus, RaTG13, SARS-CoV, WIV1, WIV16 and RsSHC014 (Fig. 1f, g, Supplementary Table 2). Consistent with the surface plasmon resonance data, S2X259 neutralized VSV pseudotypes containing $S$ of Guanxi pangolin coronavirus but with reduced potency as compared to SARS-CoV-2 (Fig. 1g, Extended Data Fig. 2a, Supplementary Table 2).

\section{Structural basis of sarbecovirus neutralization}

Three-dimensional classification of the cryo-electron microscopy data showed the presence of SARS-CoV-2 S ectodomain trimers saturated with three S2X259 Fabs bound to open RBDs swung out to various extents, for which we determined a consensus structure at $3.2 \AA$ resolution (Fig. 2a, b, Extended Data Fig. 4, Supplementary Table 3). We used local refinement to determine a 3.7 A resolution map of the region corresponding to the S2X259 variable domains and RBD. In parallel, we crystallized the S2X259 Fab-bound SARS-CoV-2 RBD in the presence of the S2H97 Fab ${ }^{26}$ and determined a 2.65 Å resolution X-ray structure (Fig. 2c-e, Supplementary Table 4).

S2X259 recognizes a glycan-free, cryptic epitope within antigenic site Ila, which was previously defined on the basis of the nonclonally related S2X35 mAb that was isolated from the same donor ${ }^{17}$. S2X259 binding requires opening of two RBDs to grant access to the Fab in the context of the S trimer (Fig. 2a, b). S2X259 contacts the RBD using both heavy and light chains, which respectively contribute two thirds and one third of the approximately $950 \AA^{2}$ paratope surface that is buried upon binding. Specifically, S2X259 uses complementary determining regions (CDRs) H1, H2, H3, L1 and L3 to contact RBD residues 369-386, which form two $\alpha$-helices and an intervening $\beta$-strand belonging to the structurally conserved RBD $\beta$-sheet, as well as the residues 404-411 mutations are shown as red spheres (PDB code 7M7W). d, e, Close-up views showing selected interactions formed between S2X259 and the SARS-CoV-2 RBD. In a-e, each SARS-CoV-2S protomer is coloured distinctly (cyan, pink and gold), and the S2X259 light and heavy chain variable domains are coloured magenta and purple, respectively. $\mathrm{N}$-linked glycans are rendered as blue spheres in $\mathbf{a}-\mathbf{c}$.

and 499-508, which form a continuous surface made up of an $\alpha$-helix and a loop followed by an $\alpha$-helix, respectively.

The S2X259 epitope is conserved in circulating SARS-CoV- 2 isolates and does not comprise prevalent RBD mutations, such as $\mathrm{S} 477 \mathrm{~N}, \mathrm{~N} 439 \mathrm{~K}$ or L452R. S2X259 also circumvents residues 417 and 484, and contacts the backbone of residue N501 but not its side chain, explaining the high-affinity binding to the B.1.1.7, B.1.351 and P.1 RBDs and potent neutralization of pseudotyped variant of concern viruses (Fig. 1b, $d$ Extended Data Fig. 2b). S2X259 broad neutralization results from the conservation of its epitope among sarbecoviruses and from the angle of approach of the Fab that circumvents the SARS-CoV N357 glycan present in all sarbecoviruses, except for SARS-CoV-2 (SARS-CoV-2 N370) (Extended Data Fig.5). The SARS-CoV-2 RBD and that of Guanxi pangolin coronavirus differ at only two positions (G504N and Y508F), and the structural data indicate that the former substitution would probably dampen $\mathrm{mAb}$ binding through steric hindrance as indicated by in vitro neutralization data (Fig. 1g).

S2X259 binding to the RBD does not affect or prevent recognition by site-I or site-IV-targeting mAbs (Extended Data Fig. 6a, b), which represent the majority of mAbs currently approved or in the clinic $^{17,22,27,28}$. Competition binding assays suggest that serum antibodies directed to the RBD antigenic site II are subdominant in individuals who have recovered from SARS-CoV-2 infection and vaccinated individuals, as compared to antibodies directed towards site I (Extended Data Fig. 6c). Therefore, S2X259 can be used in combination with other site I or site IV mAbs to maximize breadth against currently circulating and emerging SARS-CoV-2 variants, as well as against future sarbecovirus infections from animal reservoirs.

\section{S2X259 has an unusually narrow escape profile}

Using deep-mutational scanning with a yeast-display RBD mutant library $^{29}$, we exhaustively mapped mutations that escape S2X259 binding. Notably, binding was strongly reduced by only a restricted number of amino acid substitutions, as compared to previously described neutralizing $\mathrm{mAbs}^{26}$, with mutations at RBD site 504 yielding the most marked reduction in binding (Fig. 3a, Extended Data Fig. 7a-c). Indeed, substitution of G504 with most other amino acids reduced S2X259 


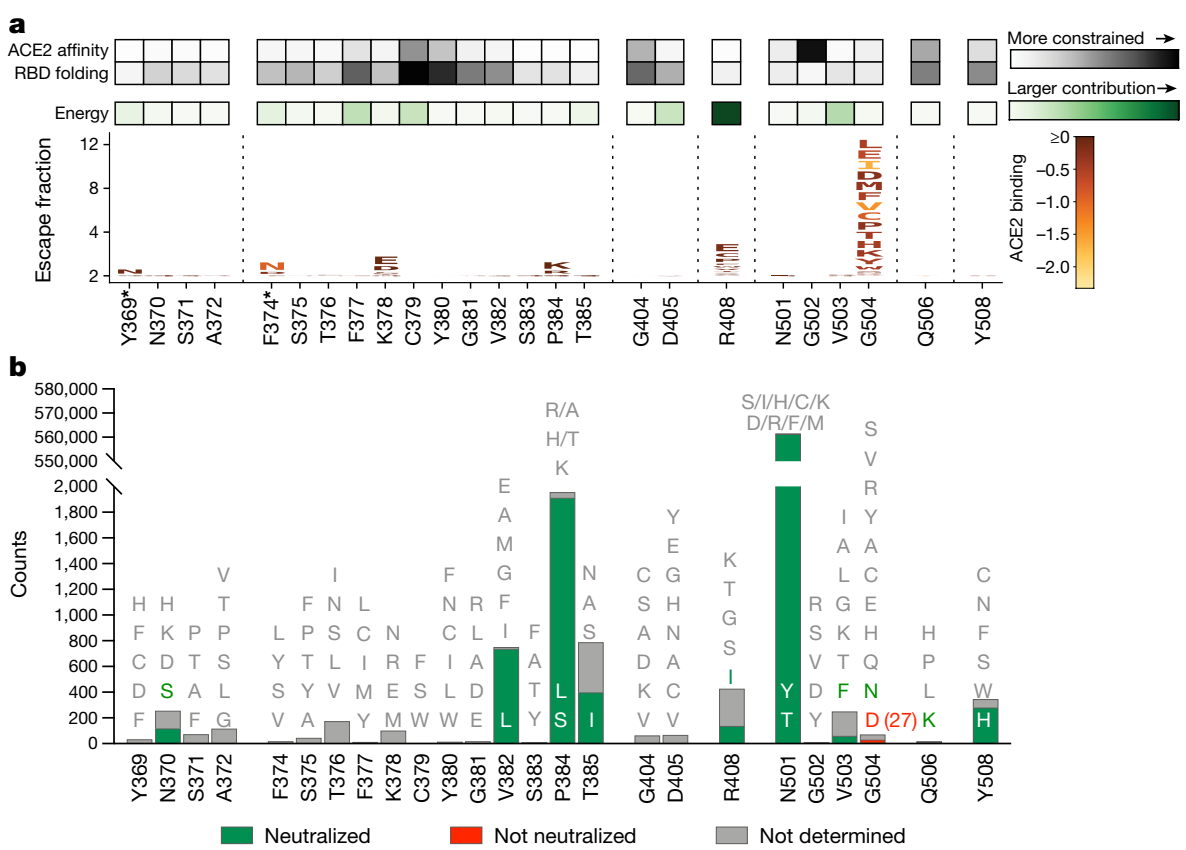

Fig. 3 | S2X259 is resilient to a broad spectrum of escape mutations. a, Complete mapping of mutations reducing S2X259 binding using yeast-displayed RBD and deep-mutational scanning. Mean mutation effect on ACE2 affinity, RBD folding and contribution to S2X259 binding of substitutions at each position in the S2X259 epitope are reported. Asterisks denote

binding compared to the wild-type (Wuhan-Hu-1) RBD, which highlights the importance of this residue for $\mathrm{mAb}$ recognition.

To further explore the functional importance of the deep-mutational scanning data, we passaged a replication-competent VSV-SARS-CoV-2 S chimera ${ }^{30,31}$ in Vero cells in the presence of S2X259 mAb. The selective pressure imposed by S2X259 led to the emergence of viral mutants containing the G504D mutation, which was the sole mutation present in each of the 18 neutralization-resistant plaques we sampled. This substitution did not impair viral fitness of the VSV-SARS-CoV-2S chimera (Extended Data Fig. 7d, e, Supplementary Table 5). Under the same conditions, S2X $35^{17}$ (which also targets antigenic site Ila) selected for seven escape mutants at five different positions (Y369S, K378E, D405N, V503D or V503G and G504D or G504S) ${ }^{26}$ (Supplementary Table 5).

The G504D substitution is very rare: it has been detected in only 27 out of 1,345,708 (0.002\%) SARS-CoV-2 genome sequences available on GISAID, as of May 2021 (Fig. 3b). Furthermore, in agreement with the deep-mutational scanning results, S2X259 efficiently neutralized VSV pseudoviruses containing S mutations located in S2X259-contact residues and found with high frequency in human isolates (Fig. 3b, Extended Data Fig. 7f). In the same analysis, we confirmed the loss of S2X259 neutralizing activity against the G504D mutant. S2X259 CDRL1 and CDRL3 contact G504 or surrounding residues, which explains why substituting a glycine with any other residue prevents mAb binding (via steric hindrance along with potential disruption of the interactions of the Fab with RBD residue D405, the latter being in close proximity to G504) (Extended Data Fig. 7g).

These data also support the hypothesis proposed above for the markedly reduced affinity and neutralization potency of S2X259 against Guanxi pangolin pseudovirus which contains the G504N mutation but maintains an aspartate at position 405 (Fig. 1g, Extended Data Figs. 2a, 7f, 8). However, S2X259 binding to clade 2S glycoproteins that bear G504D or G504E mutations (for example, those of Shanxi2011 or YN2013) was not abrogated (Fig. 1b), possibly owing to substitution at theSARS-CoV-2 equivalent position 405 with a serine and to substantial deletions in the receptor-binding motif (Extended Data Fig. 8). mutations introducing $\mathrm{N}$-linked glycosylation sites that may not be tolerated in full S. b, Frequency of mutants within the S2X259 epitope on the basis of SARS-CoV-2 genome sequences available on GISAID as of May 2021. S2X259 neutralizing activity against selected mutations is reported.

The finding that the only escape mutation for S2X259 is the rare G504D substitution suggests this mAb might have a high barrier for the emergence of SARS-CoV-2 escape mutants, which might prove essential during the next stages of the pandemic.

\section{S2X259 protects hamsters from SARS-CoV-2 challenge}

Consistent with structural data that indicates a clash between S2X259 and ACE2 for binding to the RBD (Extended Data Fig. 9a), we found that S2X259 efficiently blocked binding of SARS-CoV-2 and SARS-CoV RBDs to human ACE2 (Extended Data Fig. 9b).

In addition, S2X259 binding efficiently promoted shedding of the S1 subunit from cell-surface-expressed full-length SARS-CoV-2 S, as has previously been shown with other RBD-specific $\mathrm{mAbs}^{17,32-35}$ but not with S2M11 (which locks $S$ in the prefusion closed state) ${ }^{21}$ (Extended Data Fig. 9c). The efficient S1 shedding induced by S2X259 might be responsible for the lack of Fc $\gamma$ RIIa and Fc $\gamma$ RIIIa activation, which we used as a proxy for antibody-dependent cellular phagocytosis and antibody-dependent cellular cytotoxicity, respectively (Extended Data Fig. 9d, e). Indeed, performing the same assays using target cells that express an uncleavable prefusion stabilized SARS-CoV-2S protein (unable to release the S1 subunit), we confirmed the ability of S2X259 $\mathrm{mAb}$ to induce activation of Fc $\gamma$ RIla and Fc $\gamma$ RIIIa in these conditions (Extended Data Fig. 9f, g).

These data show that the primary mechanism of S2X259-mediated neutralization of SARS-CoV-2 and other sarbecoviruses relies on blocking viral attachment to host-cell receptors through competitive inhibition of ACE2 binding. Premature triggering of S1 subunit shedding could inactivate viruses before encountering target cells, albeit reducing activation of S2X259-mediated effector functions.

Next, we evaluated the prophylactic activity of S2X259 against challenge with the prototypic (Wuhan-Hu-1-related) SARS-CoV-2 in a Syrian hamster model ${ }^{36}$.S2X259 was administered at 1,5 and $25 \mathrm{mg}$ per kg body weight via intraperitoneal injection before intranasal challenge, and the lungs of the hamsters were collected 4 days later for the quantification 


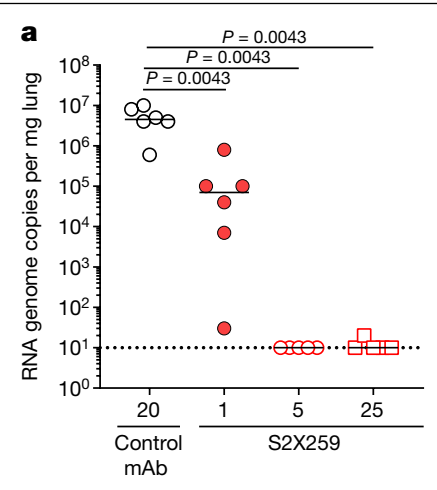

c
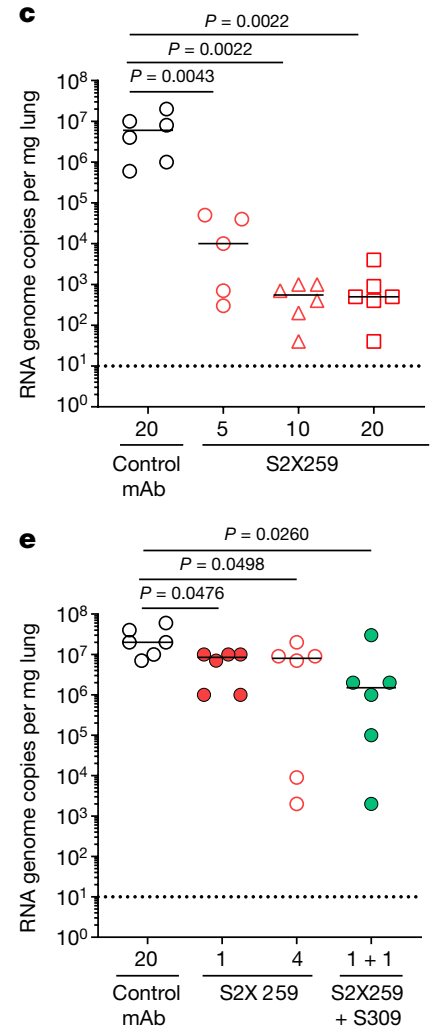
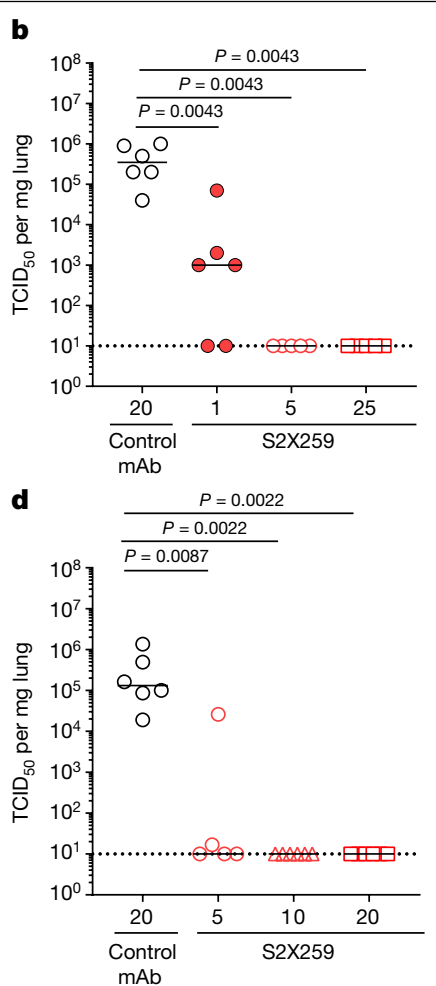

f

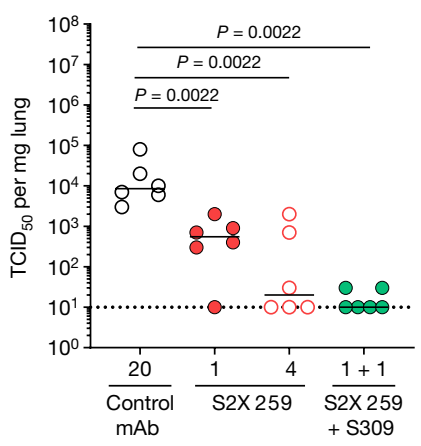

Fig. 4 | S2X259 protects hamsters challenged with prototypic and $B .1 .351$ SARS-CoV-2. a, b, Viral RNA loads (a) and replicating virus titres (50\% tissue culture infectious dose $\left.\left(\operatorname{TCID}_{50}\right)\right)(\mathbf{b})$ in the lungs of Syrian hamsters at 4 days after intranasal infection with prototypic SARS-CoV-2 (Wuhan-Hu-1-related) following prophylactic administration of S2X259 $\mathrm{mAb}$ at 1 ( $n=6$ hamsters), 5 ( $n=5$ hamsters) and 25 ( $n=5$ hamsters) $\mathrm{mg} \mathrm{kg}^{-1} . \mathbf{c}, \mathbf{d}$, Viral RNA loads (c) and replicating virus titres $\left(\mathrm{TCID}_{50}\right)(\mathbf{d})$ in the lungs of Syrian hamsters after therapeutic administration of S2X259 at 5 ( $n=5$ hamsters), 10 ( $n=6$ hamsters) and 20 ( $n=6$ hamsters) $\mathrm{mg} \mathrm{kg}^{-1} 24 \mathrm{~h}$ after infection with SARS-CoV-2 (Wuhan-Hu-1-related).e,f, Quantification of viral RNA loads (e) and replicating virus titres $\left(\mathrm{TCID}_{50}\right)(\mathbf{f})$ in the lungs of Syrian hamsters 4 days after intranasal challenge with B.1.351SARS-CoV-2 variant of concern following prophylactic administration of S2X 259 at $1 \mathrm{mg} \mathrm{kg}^{-1}$ ( $n=6$ hamsters), $4 \mathrm{mg} \mathrm{kg}^{-1}$ ( $n=6$ hamsters) and in combination with $\mathrm{S} 309\left(1+1 \mathrm{mg} \mathrm{kg}^{-1}, n=6\right.$ hamsters). Data from one independent experiment are presented. Two-tailed Mann-Whitney test was used for statistical analysis of significance.

of viral RNA and replicating virus. Despite the lack of Fc-dependent effector functions in vitro and their proposed contribution to in vivo protection against SARS-CoV-2 ${ }^{37,38}$, administration of S2X259 at $1 \mathrm{mg} \mathrm{kg}^{-1}$ reduced the amount of viral RNA and infectious virus detected in the lungs by approximately 2 and 2.5 orders of magnitude, respectively, compared to hamsters that received a control mAb (Fig. 4a, b). Furthermore, complete abrogation of virus replication was observed

in the lungs of the hamsters that were treated with S2X259 at 5 and $25 \mathrm{mg} \mathrm{kg}^{-1}$ relative to the control group (Fig. 4b). Therapeutic administration (after exposure) of S2X259 at $5 \mathrm{mg} \mathrm{kg}^{-1}$ reduced the amounts of viral RNA detected by 3 log and abrogated viral replication in the lungs of all of the hamsters but one (Fig. 4c, d). Hamsters treated with S2X259 at 10 or $20 \mathrm{mg} \mathrm{kg}^{-1}$ displayed a further approximately $1 \mathrm{log}$ reduction of viral RNA and complete abrogation of viral replication (Fig. 4c, d). Viral replication in the lungs detected at day 4 inversely correlated with serum $\mathrm{mAb}$ concentration measured at the time of infection or at day 4 after infection in prophylactic and therapeutic settings, respectively (Extended Data Fig. 10a, b).

We next assessed the prophylactic activity of S2X259 against challenge with B.1.351SARS-CoV-2 ${ }^{39}$. S2X259 at 1 and $4 \mathrm{mg} \mathrm{kg}^{-1}$, or in combination with $\mathrm{S} 309$ (each $\mathrm{mAb}$ at $1 \mathrm{mg} \mathrm{kg}^{-1}$ ), significantly decreased the amount of viral RNA detected in the lungs relative to hamsters that received a control mAb (Fig. 4e). Moreover, S2X259 at $4 \mathrm{mg} \mathrm{kg}^{-1}$ or in combination with $\mathrm{S} 309$ (each $\mathrm{mAb}$ at $1 \mathrm{mg} \mathrm{kg}^{-1}$ ) completely abrogated SARS-CoV-2 B.1.351 virus replication in most of the hamsters (Fig. 4f). As was the case for the prototypic SARS-CoV-2, the amount of infectious virus at day 4 detected in the group administered only with S2X259 also inversely correlated with serum mAb concentration measured at the time of infection (Extended Data Fig. 10c). Moreover, hamsters that received the $\mathrm{mAb}$ cocktail appeared to benefit from the additive contribution of the S309 mAb (Fig. 4f, Extended Data Fig. 10d, e).

\section{Discussion}

The large number of mutations in the S glycoprotein of SARS-CoV-2 variants of concern $n^{4,23,25}$ has been shown to abrogate or reduce the neutralization potency of several $m A$ bs and vaccine-elicited antibodies $^{5-10,40}$. Moreover, the detection of a large pool of sarbecoviruses in bats and other mammals across several continents suggests that future cross-species transmission events are likely to occur ${ }^{41-43}$. However, approved SARS-CoV-2 therapeutic agents and vaccines predominantly target or elicit immunity against immunodominant, but highly variable, epitopes and therefore have a limited efficacy against antigenic drift and genetically distinct strains with zoonotic potential ${ }^{40,44}$.

Strategies for eliciting broadly neutralizing and protective sarbecovirus antibodies targeting the RBD have recently been proposed ${ }^{18,40}$, and previous work has described mAbs that are now in the clinic with extended coverage within clade 1 sarbecoviruses ${ }^{20,22}$. The recent results from a phase III study demonstrating efficacy (that is, $85 \%$ reduction of hospitalizations or deaths) of the cross-reactive derivative of the S309 mAb (sotrovimab) paves the way for the development of additional neutralizing mAbs targeting conserved RBD epitopes that may broaden the coverage across sarbecoviruses and increase the barrier to resistance.

Here we identify the S2X259 mAb that targets the RBD antigenic site II and uniquely cross-reacts with all of the SARS-CoV-2 variants of concern that we evaluated, as well as with $30 \mathrm{~S}$ trimers or RBDs from all four sarbecovirus clades. S2X259 broadly neutralizes pseudoviruses containing S proteins from the B.1.1.7, B.1.351, P.1, B.1.427/B.1.429, and B.1.617.1 variants, and from other representative members of SARS-CoV-2 and SARS-CoV clades (clades $1 \mathrm{~b}$ and 1a, respectively). Of note, S2X259 neutralizes pseudotyped WIV1S and RsSHC014 S (clade1a) viruses, which have previously been suggested to be potential zoonotic threats to human health ${ }^{41,42}$. Moreover, S2X259 also recognizes S and RBDs from bat Southeast Asian and non-Southeast Asian sarbecovirus clades (clades 2 and 3 , respectively). The coverage of clade 2 might be particularly important, as a previous study ${ }^{16}$ has shown that exogenous protease addition to pseudoviruses from this clade resulted in detectable S-mediated entry in human cells. Furthermore, although clade 2 and clade 3 sarbecoviruses have not been reported to bind human ACE2, mutation or recombination events could lead to human ACE2 binding and entry ${ }^{16,45}$. Overall, these findings indicate that S2X259 is a 


\section{Article}

promising candidate for clinical development and could be stockpiled as part of a pandemic preparedness toolbox.

On the basis of the growing body of data demonstrating that antibodies targeting the SARS-CoV-2 RBD account for most neutralizing activity in sera from patients who have recovered from COVID-19 ${ }^{17,46}$, we propose that RBD-based vaccines could better promote elicitation of high titres of S2X259-like neutralizing antibodies, owing to the enhanced accessibility of this antigenic site compared to S-based vaccines ${ }^{40,47}$. Our serological competition data confirm that the antibody responses against the conserved site targeted by S2X $259 \mathrm{mAb}$ is subdominant in donors who have recovered from COVID-19 as well as in individuals who have received S-based mRNA vaccines. We anticipate these data will guide future efforts to develop vaccines that overcome the emergence of SARS-CoV-2 variants and that are effective against all sarbecoviruses.

\section{Online content}

Any methods, additional references, Nature Research reporting summaries, source data, extended data, supplementary information, acknowledgements, peer review information; details of author contributions and competing interests; and statements of data and code availability are available at https://doi.org/10.1038/s41586-021-03817-4.

1. Deng, X. et al. Transmission, infectivity, and neutralization of a spike L452R SARS-CoV-2 variant. Cell 184, 3426-3437 (2021).

2. Challen, R. et al. Risk of mortality in patients infected with SARS-CoV-2 variant of concern 202012/1: matched cohort study. Br. Med. J. 372, n579 (2021)

3. Davies, N. G. et al. Estimated transmissibility and impact of SARS-CoV-2 lineage B.1.1.7 in England. Science 372, eabg3055 (2021)

4. Tegally, H. et al. Detection of a SARS-CoV-2 variant of concern in South Africa. Nature 592, 438-443 (2021).

5. McCallum, M. et al. SARS-CoV-2 immune evasion by the B.1.427/B.1.429 variant of concern. Science https://doi.org/10.1126/science.abi7994 (2021).

6. Wang, $Z$. et al. mRNA vaccine-elicited antibodies to SARS-CoV-2 and circulating variants Nature 592, 616-622 (2021)

7. Collier, D. A. et al. Sensitivity of SARS-CoV-2 B.1.1.7 to mRNA vaccine-elicited antibodies. Nature 593, 136-141 (2021).

8. Wibmer, C. K. et al. SARS-CoV-2 501Y.V2 escapes neutralization by South African COVID-19 donor plasma. Nat. Med. 27, 622-625 (2021).

9. Chen, R. E. et al. Resistance of SARS-CoV-2 variants to neutralization by monoclonal and serum-derived polyclonal antibodies. Nat. Med. 27, 717-726 (2021).

10. Madhi, S. A. et al. Efficacy of the ChAdOx1 nCoV-19 Covid-19 vaccine against the B.1.351 variant. N. Engl. J. Med. 384, 1885-1898 (2021).

11. Tortorici, M. A. \& Veesler, D. Structural insights into coronavirus entry. Adv. Virus Res. 105 93-116 (2019).

12. Walls, A. C. et al. Structure, function, and antigenicity of the SARS-CoV-2 spike glycoprotein. Cell 181, 281-292 (2020).

13. Wrapp, D. et al. Cryo-EM structure of the 2019-nCoV spike in the prefusion conformation Science 367, 1260-1263 (2020).

14. Kuhn, J. H., Li, W., Choe, H. \& Farzan, M. Angiotensin-converting enzyme 2: a functional receptor for SARS coronavirus. Cell. Mol. Life Sci. 61, 2738-2743 (2004).

15. Hoffmann, M. et al. SARS-CoV-2 cell entry depends on ACE2 and TMPRSS2 and is blocked by a clinically proven protease inhibitor. Cell 181, 271-280 (2020).

16. Letko, M., Marzi, A. \& Munster, V. Functional assessment of cell entry and receptor usage for SARS-CoV-2 and other lineage B betacoronaviruses. Nat. Microbiol. 5 562-569 (2020)

17. Piccoli, L. et al. Mapping neutralizing and immunodominant sites on the SARS-CoV-2 spike receptor-binding domain by structure-guided high-resolution serology. Cell 183 1024-1042 (2020).
18. Cohen, A. A. et al. Mosaic nanoparticles elicit cross-reactive immune responses to zoonotic coronaviruses in mice. Science 371, 735-741 (2021).

19. Yuan, M. et al. A highly conserved cryptic epitope in the receptor binding domains of SARS-CoV-2 and SARS-CoV. Science 368, 630-633 (2020).

20. Rappazzo, C. G. et al. Broad and potent activity against SARS-like viruses by an engineered human monoclonal antibody. Science 371, 823-829 (2021).

21. Tortorici, M. A. et al. Ultrapotent human antibodies protect against SARS-CoV-2 challenge via multiple mechanisms. Science 370, 950-957 (2020).

22. Pinto, D. et al. Cross-neutralization of SARS-CoV-2 by a human monoclonal SARS-CoV antibody. Nature 583, 290-295 (2020).

23. Davies, N. G. et al. Increased mortality in community-tested cases of SARS-CoV-2 lineage B.1.1.7. Nature 593, 270-274 (2021).

24. Thomson, E. C. et al. Circulating SARS-CoV-2 spike N439K variants maintain fitness while evading antibody-mediated immunity. Cell 184, 1171-1187 (2021).

25. Faria, N. R. et al. Genomics and epidemiology of the P.1 SARS-CoV-2 lineage in Manaus, Brazil. Science 372, 815-821 (2021).

26. Starr, T. N. et al. SARS-CoV-2 RBD antibodies that maximize breadth and resistance to escape. Nature https://doi.org/10.1038/s41586-021-03807-6 (2021).

27. Barnes, C. O. et al. SARS-CoV-2 neutralizing antibody structures inform therapeutic strategies. Nature 588, 682-687 (2020).

28. Cathcart, A L. et al. The dual function monoclonal antibodies VIR-7831 and VIR-7832 demonstrate potent in vitro and in vivo activity against SARS-CoV-2. Preprint at https:// doi.org/10.1101/2021.03.09.434607 (2021).

29. Starr, T. N. et al. Deep mutational scanning of SARS-CoV-2 receptor binding domain reveals constraints on folding and ACE2 binding. Cell 182, 1295-1310 (2020).

30. Case, J. B. et al. Replication-competent vesicular stomatitis virus vaccine vector protects against SARS-CoV-2-mediated pathogenesis in mice. Cell Host Microbe 28, 465-474 (2020).

31. Liu, Z. et al. Identification of SARS-CoV-2 spike mutations that attenuate monoclonal and serum antibody neutralization. Cell Host Microbe 29, 477-488 (2021).

32. Huo, J. et al. Neutralization of SARS-CoV-2 by destruction of the prefusion spike. Cell Host Microbe 28, 445-454.e6 (2020).

33. Walls, A. C. et al. Unexpected receptor functional mimicry elucidates activation of coronavirus fusion. Cell 176, 1026-1039 (2019).

34. Wec, A. Z. et al. Broad neutralization of SARS-related viruses by human monoclonal antibodies. Science 369, 731-736 (2020).

35. Wrobel, A. G. et al. Antibody-mediated disruption of the SARS-CoV-2 spike glycoprotein. Nat. Commun. 11, 5337 (2020).

36. Boudewijns, R. et al. STAT2 signaling restricts viral dissemination but drives severe pneumonia in SARS-CoV-2 infected hamsters. Nat. Commun. 11, 5838 (2020).

37. Schäfer, A. et al. Antibody potency, effector function, and combinations in protection and therapy for SARS-CoV-2 infection in vivo. J. Exp. Med. 218, e20201993 (2021).

38. Winkler, E. S. et al. Human neutralizing antibodies against SARS-CoV-2 require intact Fc effector functions and monocytes for optimal therapeutic protection. Cell 184 1804-1820 (2021).

39. Abdelnabi, R. et al. Comparing infectivity and virulence of emerging SARS-CoV-2 variants in Syrian hamsters. EBioMedicine 68, 103403 (2021).

40. Walls, A. C. et al. Elicitation of broadly protective sarbecovirus immunity by receptor-binding domain nanoparticle vaccines. Preprint at https://doi.org/10.1101/2021.03.15.435528 (2021).

41. Menachery, V. D. et al. A SARS-like cluster of circulating bat coronaviruses shows potential for human emergence. Nat. Med. 21, 1508-1513 (2015).

42. Menachery, V. D. et al. SARS-like WIV1-CoV poised for human emergence. Proc. Natl Acad. Sci. USA 113, 3048-3053 (2016)

43. Ge, X. Y. et al. Isolation and characterization of a bat SARS-like coronavirus that uses the ACE2 receptor. Nature 503, 535-538 (2013)

44. Martinez, D. R. et al. Chimeric spike mRNA vaccines protect against Sarbecovirus challenge in mice. Science https://doi.org/10.1126/science.abi4506 (2021).

45. Makarenkov, V., Mazoure, B., Rabusseau, G. \& Legendre, P. Horizontal gene transfer and recombination analysis of SARS-CoV-2 genes helps discover its close relatives and shed light on its origin. BMC Ecol. Evol. 21, 5 (2021).

46. Greaney, A. J. et al. Comprehensive mapping of mutations in the SARS-CoV-2 receptor-binding domain that affect recognition by polyclonal human plasma antibodies. Cell Host Microbe 29, 463-476 (2021).

47. Walls, A. C. et al. Elicitation of potent neutralizing antibody responses by designed protein nanoparticle vaccines for SARS-CoV-2. Cell 183, 1367-1382 (2020)

Publisher's note Springer Nature remains neutral with regard to jurisdictional claims in published maps and institutional affiliations.

(c) The Author(s), under exclusive licence to Springer Nature Limited 2021 


\section{Methods}

\section{Cell lines}

Cell lines used in this study were obtained from ATCC (HEK293T, Vero and Vero E6), ThermoFisher Scientific (ExpiCHO-S cells, FreeStyle 293-F cells and Expi293F cells) or were generated via lentiviral transduction (ExpiCHO-S and HEK293T expressing ACE2 (HEK293T-ACE2)). None of the cell lines used was authenticated. Cell lines were routinely tested for mycoplasma contamination.

\section{Sample donors}

Samples from an individual who had recovered from COVID-19 (designated as donor $X$ (a 52-year-old man)) was obtained 75 days after symptom onset under study protocols approved by the local Institutional Review Boards (Canton Ticino Ethics Committee, Switzerland). Samples from additional nine individuals who had recovered from COVID-19 and nine vaccinated individuals were obtained under study protocols approved by the Canton Ticino Ethics Committee, Switzerland and the Ethical committee of Luigi Sacco Hospital, Milan. Samples from individuals who had recovered from COVID-19 were collected between 14 and 75 days after symptom onset; samples from vaccinated individuals were collected between 13 and 20 days after the second dose of an mRNA-based SARS-CoV-2 vaccine. All donors provided written informed consent for the use of blood and blood components (such as peripheral blood mononuclear cells (PBMCs), sera or plasma).

\section{Isolation of PBMCs, plasma and sera}

PBMCs were isolated from blood draw performed using tubes prefilled with heparin, followed by Ficoll density gradient centrifugation. PBMCs were either used freshly along SARS-CoV-2S-protein-specific memory B cell sorting or stored in liquid nitrogen for later use. Sera were obtained from blood collected using tubes containing clot activator, followed by centrifugation and stored at $-80^{\circ} \mathrm{C}$.

\section{B cell isolation and recombinant $\mathrm{S2X} 259 \mathrm{mAb}$ production}

Starting from freshly isolated PBMCs or upon cells thawing, B cells were enriched by staining with CD19 PE-Cy7 and incubation with anti-PE beads, followed by positive selection using LS columns. Enriched B cells were stained with anti-IgM, anti-IgD, anti-CD14 and anti-IgA, all PE labelled, and prefusion SARS-CoV-2 $\mathrm{S}$ with a biotinylated avi tag conjugated to streptavidin Alexa-Fluor 647 (Life Technologies). SARS-CoV-2S-specific IgG ${ }^{+}$memory B cells were sorted by flow cytometry via gating for PE-negative and Alexa-Fluor 647-positive cells. Sorted IgG $\mathrm{Ig}^{+}$memory B cells were seeded in 384-well microtitre plates on a monolayer of mesenchymal stromal cells at 0.5 cells per well in the presence CpG2006, IL-2, IL-6 and IL-21, as previously described $^{48}$. After 7 days, the supernatants were screened for binding to SARS-CoV-2 RBD and prefusion $S$ as well as for neutralization activity using a high-throughput VSV SARS-CoV-2S-based microneutralization assay. Selection of the antibody of interest in secondary screening was based on reactivity to a panel of sarbecovirus RBDs as well as on the neutralizing activity. Antibody $\mathrm{VH}$ and $\mathrm{VL}$ sequences were obtained by RT-PCR and mAbs were expressed as recombinant human Fab fragment or as IgG1 (G1m3 allotype). ExpiCHO cells were transiently transfected with heavy and light chain expression vectors as previously described ${ }^{22}$.

Affinity purification was performed on ÄKTA Xpress FPLC (Cytiva) operated by UNICORN software version 5.11 (build 407) using HiTrap Protein A columns (Cytiva) for full length human and hamster mAbs and CaptureSelect CH1-XL MiniChrom columns (ThermoFisher Scientific) for Fab fragments, using PBS as mobile phase. Buffer exchange to the appropriate formulation buffer was performed with a HiTrap Fast desalting column (Cytiva). The final products were sterilized by filtration through $0.22-\mu \mathrm{m}$ filters and stored at $4{ }^{\circ} \mathrm{C}$.
Sarbecovirus sequence analysis and SARS-CoV-2 mutant counts Alignment and phylogenetic tree of the strains within the Sarbecovirus subgenus was generated using MEGAX and CLC Main work bench 21.0.3 (Qiagen). The following sequence was retrieved from GISAID and NCBI: A021 (AAV97986.1). The viral sequences were obtained from GISAID EpiCoV project (https://www.gisaid.org/). Analysis was performed on sequences submitted to GISAID up to 2 April 2021. The S protein sequences were either obtained directly from the protein dump provided by GISAID or, for the latest submitted sequences that were not yet incorporated in the protein dump on the day of data retrieval, from the genomic sequences with exonerate ${ }^{49} 22.4 .0$-haf93ef1_3 (https://quay. io/repository/biocontainers/exonerate?tab=tags) using protein to DNA alignment with parameters - $m$ protein $2 \mathrm{dna}$--refine full --minintron 999999 --percent 20 and using accession YP_009724390.1 as a reference. Multiple sequence alignment of all human $\mathrm{S}$ proteins was performed with mafft ${ }^{50} 7.475-\mathrm{h} 516909 a+0$ (https://quay.io/repository/biocontainers/mafft?tab=tags) with parameters --auto--reorder --keeplength --addfragments using the same reference as above. S sequences that contained more than $10 \%$ ambiguous amino acids or that were less than $80 \%$ of the canonical protein length were discarded. A total of 923,686 sequences were used for analysis. Variants were then extracted as compared to the reference with R 4.0.2 (https:// www.r-project.org/) using Biostrings 2.56.0.

\section{Binding to cell-surface-expressed sarbecovirus $S$ proteins by flow cytometry}

ExpiCHO-S cells were seeded at $6 \times 10^{6}$ cells per $\mathrm{ml}$ in a volume of $5 \mathrm{ml}$ in a 50-ml bioreactor. S-encoding plasmids HKU3 (QND76020.1), Rs3367 (AGZ48818.1), YN2013 (AIA62330.1), Rs4874 (ATO98205.1), Rs4255 (ATO98193.1), Rs4231 (ATO98157.1), Rs4084 (ATO98132.1), ZXC21(AVP78042.1),ZC45 (AVP78031.1), Rp/Shaanxi2011 (AGC74165.1), Rm1/2004 (ABD75332.1), Rf1-2004 (ABD75323.1), BM48-31/ BGR/2008 (YP_003858584.1), RaTG13 (QHR63300.2), PC4-127 (AAU93318.1), SARS-CoV-2 (YP_009724390.1), LYRa3 (AHX37569.1), Cp/Yunnan2011 (AGC74176.1), SARS-CoV Urbani (AAP13441.1), As6526 (ATO98108.1), BtKY72 (APO40579.1), RmYN02 (EPI_ISL_412977), Pangolin_Guangdong-2019 (EPI_ISL_410721), Pangolin-Guanxi-2017 (EPI_ ISL_410539) and HKU3-12 (ADE34812.1) were diluted in cold OptiPRO SFM (Life Technologies, 12309-050), mixed with ExpiFectamine CHO Reagent (Life Technologies, A29130) and added to the cells. Transfected cells were then incubated at $37^{\circ} \mathrm{C}$ with $8 \% \mathrm{CO}_{2}$ with an orbital shaking speed of 250 r.p.m. (orbital diameter of $25 \mathrm{~mm}$ ) for $42 \mathrm{~h}$. Transiently transfected ExpiCHO cells were collected and washed two times in wash buffer (PBS 2\% FBS, 2 mM EDTA). Cells were counted and distributed into round-bottom 96-well plates (Corning, 3799) and incubated with S2X259 mAb serial dilutions from $50 \mu \mathrm{g} \mathrm{ml}^{-1}$. Alexa Fluor647-labelled goat anti-human IgG secondary antibody (Jackson Immunoresearch, 109-606-098) was prepared at $2 \mathrm{\mu g} \mathrm{ml}^{-1}$ and added onto cells after two washing steps. Cells were then washed twice and resuspended in wash buffer for data acquisition at ZE5 cytometer (Biorad).

\section{Recombinant protein production}

Wild-type SARS-CoV-2 RBD (with N-terminal signal peptide and 'ETGT', and C-terminal $8 \times \mathrm{His} \mathrm{tag}$ ) was expressed in Expi293F cells at $37^{\circ} \mathrm{C}$ and $8 \% \mathrm{CO}_{2}$ in the presence of $10 \mu \mathrm{M}$ kifunensine. Transfection was performed using the ExpiFectamine 293 Transfection Kit (Thermo Fisher Scientific). Cell culture supernatant was collected four days after transfection and supplemented with $10 \times \mathrm{PBS}$ to a final concentration of $2.5 \times \mathrm{PBS}$ ( $342.5 \mathrm{mM} \mathrm{NaCl}, 6.75 \mathrm{mM} \mathrm{KCl}$ and $29.75 \mathrm{mM}$ phosphates). For crystallization, wild-type SARS-CoV-2 RBD was purified using a 5-ml HisTALON superflow cartridge (Takara Bio) followed by size-exclusion chromatography using a Superdex 200 Increase 10/300 GL column pre-equilibrated in $20 \mathrm{mM}$ Tris- $\mathrm{HCl}$ pH 7.5, $150 \mathrm{mM} \mathrm{NaCl}$. RBD was deglycosylated with EndoH and mixed with a 1.3-fold molar 
excess of S2X259 Fab and S2H97 Fab. The complex was purified on a Superdex 200 Increase $10 / 300$ GL column pre-equilibrated with $20 \mathrm{mM}$ Tris- $\mathrm{HCl}$ pH 7.5, $150 \mathrm{mM} \mathrm{NaCl}$. SARS-CoV-2 S hexapro protein, used for cryo-electron microscopy (cryo-EM) single particle studies, was expressed and purified as previously described ${ }^{21}$.

\section{ELISA}

Ninety-six half area well-plates (Corning, 3690) were coated overnight at $4{ }^{\circ} \mathrm{C}$ with $25 \mu$ l of sarbecovirus RBD proteins WIV1 (AGZ48831.1), Anlong-112 (ARI44804.1), YN2013 (AIA62330.1), SC2018 (QDF43815.1), ZC45 (AVP78031.1), Rp/Shaanxi2011 (AGC74165.1), BM48-31/BGR/2008 (YP_003858584.1), RaTG13 (QHR63300.2), SARS-CoV-2 (YP_009724390.1), SARS-CoV Urbani (AAP13441.1), BtKY72 (APO40579.1), Pangolin_Guangdong-2019 (EPI_ISL_410721) and SARS-CoV-2 RBD mutants, prepared at $5 \mu \mathrm{g} \mathrm{ml}^{-1}$ in PBS pH 7.2. Plates were then blocked with PBS 1\% BSA (Sigma-Aldrich, A3059) and subsequently incubated with $\mathrm{mAb}$ serial dilutions for $1 \mathrm{~h}$ at room temperature. After 4 washing steps with PBS 0.05\% Tween 20 (PBS-T) (Sigma-Aldrich, 93773), goat anti-human IgG secondary antibody (Southern Biotech, 2040-04) was added and incubated for $1 \mathrm{~h}$ at room temperature. Plates were then washed four times with PBS-T and 4-nitrophenyl phosphate (pNPP, Sigma-Aldrich, 71768) substrate was added. After 30 min incubation, absorbance at $405 \mathrm{~nm}$ was measured by a plate reader (Biotek) and data were plotted using Prism GraphPad 9.1.0.

\section{S2X259 blockade-of-binding to RBD}

S2X259 and S2H14 mAbs were biotinylated using EZ-Link NHS-PEG solid phase biotinylation kit (ThermoFisher Scientific) and binding to RBD was tested to set optimal concentration to be used in the assay after sample desalting using Zeba Spin Desalting Columns (ThermoFisher Scientific). Half-area 96-well-plates were coated overnight at $4{ }^{\circ} \mathrm{C}$ with SARS-CoV-2 RBD-mouse Fc Tag diluted at $1 \mu \mathrm{g} \mathrm{ml}^{-1}$ in PBS. After a blocking step with Blocker Casein (ThermoFisher Scientific), serial plasma dilutions in Blocker Casein were incubated $30 \mathrm{~min}$ at room temperature. Biotinylated S2X259 or S2H14 were added at a concentration achieving $80 \%$ of maximal binding and the mixture was incubated for $30 \mathrm{~min}$ at room temperature. Alkaline-phosphatase conjugated streptavidin (Jackson ImmunoResearch) was diluted at $0.5 \mu \mathrm{g} \mathrm{ml}^{-1}$ in Blocker Casein and added on plates previously washed 4 times with PBS 0.05\% Tween 20. After $45 \mathrm{~min}$ incubation, plates were washed and 4-nitrophenyl phosphate substrate incubated for $1 \mathrm{~h}$ at room temperature. Absorbance at $405 \mathrm{~nm}$ was measured and percentage of inhibition was calculated as follows: (1 - (optical density (OD) sample - OD negative control $) /($ OD positive control - OD negative control $)) \times 100$.

\section{MLV-based pseudotyped virus production and neutralization}

To generate SARS-CoV-2S MLV pseudotyped virus, HEK293T cells were seeded in $10-\mathrm{cm}$ dishes in DMEM supplemented with $10 \% \mathrm{FBS}$. The next day cells were transfected with a SARS-CoV-2S glycoprotein-encoding plasmid containing the D19 C-terminal truncation ${ }^{51}$, an MLV Gag-Pol packaging construct and the reporter vector pTG-Luc, using the X-tremeGENE HP DNA transfection reagent (Roche) according to the manufacturer's instructions. Cells were then incubated at $37^{\circ} \mathrm{C}$ with $5 \% \mathrm{CO}_{2}$ for $72 \mathrm{~h}$. Supernatant was collected and cleared from cellular debris by centrifugation at $400 \mathrm{~g}$, and stored at $-80^{\circ} \mathrm{C}$.

For neutralization assays, Vero E6 cells were seeded into white 96 -well plates (PerkinElmer) at 20,000 cells per well and cultured overnight at $37^{\circ} \mathrm{C}$ with $5 \% \mathrm{CO}_{2}$ in $100 \mu \mathrm{l}$ DMEM supplemented with $10 \% \mathrm{FBS}$ and $1 \%$ penicillin-streptomycin. The next day, MLV-SARS-CoV-2 pseudovirus was activated with $10 \mathrm{\mu g} \mathrm{ml}^{-1}$ TPCK-treated trypsin (Worthington Biochem) for $1 \mathrm{~h}$ at $37^{\circ} \mathrm{C}$. Recombinant antibodies at various concentrations were incubated with activated pseudovirus for $1 \mathrm{~h}$ at $37^{\circ} \mathrm{C}$. The Vero E6 cells were then washed with DMEM, and $50 \mu$ of pseudovirus-mAbs mixes were added and incubated for $2 \mathrm{~h}$ at $37^{\circ} \mathrm{C}$ with $5 \% \mathrm{CO}_{2}$. After incubation, $50 \mu \mathrm{l}$ of DMEM containing $20 \% \mathrm{FBS}$ and $2 \%$ penicillin-streptomycin were added and the cells were incubated $48 \mathrm{~h}$ at $37^{\circ} \mathrm{C}$ with $5 \% \mathrm{CO}_{2}$. Following these $48 \mathrm{~h}$ of infection, culture medium was removed from the cells and $50 \mu \mathrm{l}$ per well of Bio-Glo (Promega) diluted 1:2 with PBS with $\mathrm{Ca}^{2+} \mathrm{Mg}^{2+}$ (Thermo Fisher) was added to the cells and incubated in the dark for $15 \mathrm{~min}$ before reading on a Synergy H1 Hybrid Multi-Mode plate reader (Biotek). Measurements were done in duplicate, Relative light unit (RLU) values were converted to percentage of neutralization and plotted with a nonlinear regression curve fit in GraphPad Prism 9.1.0.

\section{VSV-based pseudotype virus production and neutralization} assay

SARS-CoV-2 S (CAD0240757.1), RaTG13 S (QHR63300.2), Pangolin-Guangdong S (QLR06867.1), Pangolin-Guanxi S (QIA48623.1), SARS-CoV S (YP 009825051.1), WIV1 S (AGZ48831.1), WIV16 S (ALK02457.1), RsSHCO14S (AGZ48806.1), the variant of concern B.1.429 S (QTC60823) and the variants of concern, N437K, Y453F, B.1.1.7 S, B.1.351S and P.1S with their corresponding mutations inserted in the SARS-CoV-2S (CAD0240757.1) backbone were used to pseudotype VSV. Pseudotyped viruses were prepared using $293 \mathrm{~T}$ cells seeded in $10-\mathrm{cm}$ dishes. In brief, cells in DMEM supplemented with $10 \%$ FBS, $1 \%$ penicillin-streptomycin were transfected with the plasmid encoding for the corresponding S glycoprotein using lipofectamine 2000 (Life Technologies) following manufacturer's indications. One day after transfection, cells were infected with VSV (G* $\Delta \mathrm{G}$-luciferase) and after $2 \mathrm{~h}$, infected cells were washed 4 times with DMEM before adding medium supplemented with anti-VSV-G antibody (I1- mouse hybridoma supernatant diluted 1 to 50, from CRL-2700, ATCC). Particles were collected $18 \mathrm{~h}$ after inoculation, clarified from cellular debris by centrifugation at 2,000 $\mathrm{g}$ for $5 \mathrm{~min}$ and concentrated 10 times using a 30-kDa cut-off membrane and used for neutralization experiments, aliquoted and frozen at $-80^{\circ} \mathrm{C}$ until use in neutralization experiments.

For neutralization, stable 293 T cells expressing ACE2 ${ }^{52}$ in DMEM supplemented with $10 \% \mathrm{FBS}, 1 \%$ penicillin-streptomycin were seeded at 40,000 cells per well into clear-bottom white-walled 96 -well plates and cultured overnight at $37^{\circ} \mathrm{C}$. Twelve-point threefold serial dilutions of the corresponding $\mathrm{mAb}$ were prepared in DMEM and pseudotyped VSV viruses were added 1:1 to each mAb dilution in the presence of anti-VSV-G antibody from I1- mouse hybridoma supernatant diluted 50 times. After $45 \mathrm{~min}$ incubation at $37^{\circ} \mathrm{C}, 40 \mu \mathrm{l}$ of the mixture was added to the cells and $2 \mathrm{~h}$ after infection, $40 \mu \mathrm{l}$ DMEM was added to the cells. After $17-20 \mathrm{~h} 50 \mu \mathrm{l}$ per well of One-Glo-EX substrate (Promega) was added to the cells and incubated in the dark for 5-10 min before reading on a Varioskan LUX plate reader (ThermoFisher). Measurements were done in duplicate with two independent productions of pseudotyped viruses and RLU values were converted to percentage of neutralization and plotted with a nonlinear regression curve fit in GraphPad Prism 9.1.0.

\section{Generation of VSV SARS-CoV-2 S pseudotypes from a selection of circulating variants}

Replication-defective VSV pseudovirus expressing SARS-CoV-2S protein were generated as previously described ${ }^{53}$ with some modifications. Plasmids encoding the SARS-CoV-2 S glycoprotein corresponding to the variants of concern used to produce SARS-CoV-2-VSV were obtained using a multispep overlap extension PCR protocol ${ }^{5,7}$. In brief, the mutations of the different variant of concern lineages were encoded on each primer pair used to amplify sequential, overlapping fragments of the SARS-CoV-2 S encoding DNA sequence from a pCDNA_SARS-CoV-2_S D19 plasmid encoding the $C$-terminally truncated $S$ protein that has been shown to be better expressed at the cell ${ }^{30}$. The amplified overlapping fragments were separated on agarose gel and purified using Illustra GFX PCR DNA and Gel Band Purification Kit (Merck KGaA). The purified overlapping fragments were joined by performing 10 cycles of PCR without the addition of primers, followed by 30 PCR cycles using 
a single pair of external primers carrying 5 ' overhangs homologous to the vector backbone. The final PCR product was then cloned into the pCDNA3 vector using the Takara In-fusion HD cloning kit following manufacturer's instructions.

Lenti-X 293T cells (Takara, 632180) were seeded in $10-\mathrm{cm}$ dishes at a density of $5 \times 10^{6}$ cells per $\mathrm{cm}^{2}$ and the following day transfected with $10 \mu \mathrm{g}$ of S expression plasmid with TransIT-Lenti (Mirus, 6600) according to the manufacturer's instructions. One day after transfection, cells were infected with VSV-luc (VSV-G) (Kerafast, EH1020-p.m.) for $1 \mathrm{~h}$, rinsed three times with PBS, then incubated for an additional $24 \mathrm{~h}$ in complete medium at $37^{\circ} \mathrm{C}$. The cell supernatant was clarified by centrifugation, filtered $(0.45 \mu \mathrm{m})$, aliquoted and frozen at $-80^{\circ} \mathrm{C}$.

For VSV pseudovirus neutralization assays, Vero-E6 cells were grown in DMEM supplemented with $10 \%$ FBS and seeded into CulturePlate-96, White Opaque 96-well Microplate (PerkinElmer) at a density of $2 \times 10^{4}$ cells per well. The next day, mAbs were serially diluted in prewarmed complete medium, mixed at 1:1 ratio with pseudovirus and incubated for $1 \mathrm{~h}$ at $37^{\circ} \mathrm{C}$ in round-bottom polypropylene plates. Medium from cells was aspirated and $50 \mu \mathrm{l}$ of virus $-\mathrm{mAb}$ complexes were added to cells and then incubated for $1 \mathrm{~h}$ at $37^{\circ} \mathrm{C}$. An additional $100 \mu \mathrm{l}$ of prewarmed complete medium was then added on top of complexes and cells incubated for an additional $16-24 \mathrm{~h}$. Conditions were tested in duplicate wells.

Virus-mAb-containing medium was then aspirated from cells and $50 \mu \mathrm{l}$ of a 1:2 dilution of SteadyLite Plus (Perkin Elmer) in PBS with $\mathrm{Ca}^{2+} \mathrm{Mg}^{2+}$ was added to cells. Plates were incubated for $10 \mathrm{~min}$ at room temperature and then analysed on the Synergy H1 Hybrid Multi-Mode reader (Biotek). Measurements were done in two independent experiments and RLU values were converted to percentage of neutralization and plotted with a nonlinear regression curve fit with an upper constraint equal to 100 and a lower constraint equal to 0 in Prism (version 9.1.0). Half-maximal inhibitory concentration $\left(\mathrm{IC}_{50}\right)$ values across biological replicates are presented as arithmetic mean \pm s.d.

\section{Neutralization of SARS-CoV-2 B.1.351 pseudotyped VSV by S2X259 + S309 antibody cocktail}

Vero E6 cells were plated into white 96-well plates (PerkinElmer) at 20,000 cells per well and cultured overnight at $37^{\circ} \mathrm{C} 5 \% \mathrm{CO}_{2}$ in $100 \mu \mathrm{l}$ culture medium (DMEM $10 \%$ FBS $1 \%$ penicillin-streptomycin). The next day, S2E12 and S309 antibodies were serially diluted in infection medium (DMEM $10 \%$ heat-inactivated FBS $1 \%$ penicillin-streptomycin) and mixed in a matrix format before incubation with SARS-CoV-2 B.1.351 pseudovirus (multiplicity of infection (MOI) 0.1 ) at $37^{\circ} \mathrm{C} 5 \% \mathrm{CO}_{2}$ for $1 \mathrm{~h}$. Cell culture medium was aspirated and $50 \mu \mathrm{l}$ of mAbs-pseudovirus mix added onto cells. After incubation at $37^{\circ} \mathrm{C} 5 \% \mathrm{CO}_{2}$ for $1 \mathrm{~h}, 100 \mu \mathrm{l}$ of infection medium was added to cell and incubation continued for the following $20 \mathrm{~h}$. For results read out, medium was removed and 50 $\mu \mathrm{l}$ per well of Steadylite PLUS (PerkinElemer) 1:2 diluted in $\mathrm{PBS}+\mathrm{Ca}^{2+}$ and $\mathrm{Mg}^{2+}$ was added and incubated $15 \mathrm{~min}$ at room temperature in the dark. Luminescence signals were measured using a Synergy $\mathrm{H} 1$ reader (Biotek) with 1-s integration time. Data were analysed using MacSynergy II and synergy plots obtained with $99.9 \%$ confidence were used for graphical elaboration with GraphPad Prism 9.1.0.

\section{Neutralization of authentic SARS-CoV-2-Nluc virus}

Neutralization of authentic SARS-CoV-2 by entry-inhibition assay was determined using SARS-CoV-2-Nluc, an infectious clone of SARS-CoV-2 (based on strain 2019-nCoV/USA_WA1/2020) that encodes nanoluciferase in place of the viral ORF7 and demonstrated comparable growth kinetics to wild-type virus ${ }^{54}$. Vero E6 cells were seeded into black-walled, clear-bottom 96 -well plates at $2 \times 10^{4}$ cells per well and cultured overnight at $37^{\circ} \mathrm{C}$. The next day, 9-point fourfold serial dilutions of $m A b s$ were prepared in infection medium (DMEM $+10 \% \mathrm{FBS}$ ). SARS-CoV-2-Nluc was diluted in infection medium at a final MOI of 0.01 plaque-forming units per cell, added to the mAb dilutions and incubated for $30 \mathrm{~min}$ at $37^{\circ} \mathrm{C}$. Medium was removed from the Vero E6 cells, $\mathrm{mAb}$-virus complexes were added and incubated at $37^{\circ} \mathrm{C}$ for $24 \mathrm{~h}$. Medium was removed from the cells, Nano-Glo luciferase substrate (Promega) was added according to the manufacturer's recommendations, incubated for $10 \mathrm{~min}$ at room temperature and the luciferase signal was quantified on a VICTOR Nivo plate reader (Perkin Elmer).

\section{Affinity determination by surface plasmon resonance}

Surface plasmon resonance binding measurements were performed using a Biacore T200 instrument, running buffer was Cytiva HBS-EP+ (pH 7.4), and all measurements were performed at $25^{\circ} \mathrm{C}$. For Fab binding, anti-AviTag pAb (for capturing S proteins) or StrepTactin XT (for capturing RBDs) were covalently immobilized on CM5 chips. S2X259 Fab concentrations were $11,33,100$, and 300 nM run as single-cycle kinetics. Double reference-subtracted data were fit to a 1:1 binding model using Biacore Evaluation software, which yields an 'apparent $\mathrm{KD}^{\prime}\left(K_{\mathrm{D}, \mathrm{app}}\right)$ for the S-binding data because the kinetics also reflect $\mathrm{S}$ conformational dynamics. For SARS-CoV-2S the dissociation rate was too slow to fit, so the $K_{\mathrm{D} \text {,app }}$ is reported as an upper limit. The $K_{\mathrm{D}}$ above $1 \mu \mathrm{M}$ is approximate and was determined from a fit in which $R_{\max }$ was set to a constant based on theoretical $R_{\max }$.

\section{Affinity determination using biolayer interferometry}

Biotinylated RBD (wild type, N501Y, K417N-E484K-N501Y, or K417T-E484K-N501Y) were immobilized at $5 \mathrm{ng}^{-1} \mathrm{l}^{-1}$ in undiluted $10 \mathrm{X}$ Kinetics Buffer (Pall) to SA sensors until a load level of $1.1 \mathrm{~nm}$. A 1:3 dilution series of Fab in undiluted kinetics buffer starting at $10 \mathrm{nM}$ was used for 600 s association before 600 s dissociation to determine proteinprotein affinity. The data were baseline-subtracted and the plots fitted using the Pall FortéBio/Sartorius analysis software (version 12.0). Data were plotted in Prism 9.1.0.

\section{Competition assay by biolayer interferometry}

Biolayer interferometry was used to assess S2X259 competition with S309 and S2E12 using an Octet Red96 (ForteBio). All reagents were prepared in kinetics buffer (PBS 0.01\% BSA) at the indicated concentrations. His-tagged SARS-CoV-2 RBD was prepared at $8 \mu \mathrm{g} \mathrm{ml}^{-1}$ and loaded on prehydrated anti-penta-HIS biosensors (Sartorius) for $2.5 \mathrm{~min}$. Biosensors were then moved into a solution containing S2X259 mAb and association recorded for $7 \mathrm{~min}$. A second association step was subsequently performed into S2X259 (as control), S309 and S2E12 mAbs solutions at $20 \mu \mathrm{g} \mathrm{ml}^{-1}$ and recorded for $7 \mathrm{~min}$. Response values were exported and plotted using GraphPad Prism 9.1.0.

\section{Cell-surface mAb-mediated S1 shedding}

CHO cells stably expressing wild-type SARS-CoV-2S were resuspended in wash buffer (PBS 1\% BSA, 2 mM EDTA) and treated with $10 \mu \mathrm{g} \mathrm{ml}^{-1}$ TPCK-trypsin (Worthington Biochem) for $30 \mathrm{~min}$ at $37^{\circ} \mathrm{C}$. Cells were then washed and distributed into round bottom 96 -well plates $(90,000$ cells per well). S2X259 was added to cells at $15 \mu \mathrm{g} \mathrm{ml}^{-1}$ final concentration for $180 \mathrm{~min}$ at $37^{\circ} \mathrm{C}$. Cells were collected at different time points $(5,30,60,120$ and $180 \mathrm{~min})$, washed with wash buffer at $4{ }^{\circ} \mathrm{C}$, and incubated with $1.5 \mu \mathrm{g} \mathrm{ml}^{-1}$ secondary goat anti-human IgG, Fc fragment specific (Jackson ImmunoResearch) on ice for $20 \mathrm{~min}$. Cells were washed and resuspended in wash buffer and analysed with ZE5 FACS (Biorad).

\section{Measurement of Fc-effector functions}

S2X259-dependent activation of human FcyRIIla was performed with a bioluminescent reporter assay. ExpiCHO cells transiently expressing full-length wild-type SARS-CoV-2S (target cells) or full-length prefusion stabilized SARS-CoV-2S, which contains the $2 \mathrm{P}$ mutation and S1/S2 furin cleavage site mutation (RRARS to SGAG) as previously described ${ }^{12}$, were incubated with different amounts of mAbs. After a 15-min incubation, Jurkat cells stably expressing Fc $\gamma$ RIIIa receptor (V158 variant) or Fc $\gamma$ RIIa receptor (H131 variant) and NFAT-driven luciferase gene (effector cells) 
were added at an effector to target ratio of 6:1 for Fc $\mathrm{R}$ RIIIa and 5:1 for Fc $\gamma$ RIIa. Signalling was quantified by the luciferase signal produced as a result of NFAT pathway activation. Luminescence was measured after $20 \mathrm{~h}$ of incubation at $37^{\circ} \mathrm{C}$ with $5 \% \mathrm{CO}_{2}$ with a luminometer using the Bio-Glo Luciferase Assay Reagent according to the manufacturer's instructions (Promega).

\section{In vivo $m A b$ testing using a Syrian hamster model}

KU LEUVEN R\&D has developed and validated a SARS-CoV-2 Syrian hamster infection mode ${ }^{36,39}$. The SARS-CoV-2 (BetaCov/Belgium/GHB03021/2020-EPI ISL 109 407976|2020-02-03), which is most closely related to the prototypic Wuhan-Hu-12019-nCoV (GenBank accession number MN908947.3) strain, and B.1.351 (hCoV10519/Belgium/rega1920/2021; EPI_ISL_896474, 2021-01-11) isolates used in this study were recovered from nasopharyngeal swabs taken from an asymptomatic returning traveller with RT-qPCR-confirmed SARS-CoV-2 infection in February 2020, and from a patient with respiratory symptoms in January 2021, respectively. A close relatedness with the prototypic Wuhan-Hu-12019SARS-CoV-2 and with B.1.351 lineage was confirmed by sequencing and phylogenetic analysis. Infectious viruses were isolated by serial passaging on Vero E6 cells and passage 6 for SARS-CoV-2 Wuhan-Hu-1 and passage 2 for B.1.351 viruses were used for the study. The titre of the virus stock was determined by end-point dilution on Vero E6 cells by a previously published method ${ }^{55}$. This work was conducted in the high-containment $\mathrm{A} 3$ and BSL3+ facilities of the KU Leuven Rega Institute (3CAPS) under licences AMV 30112018 SBB 21920180892 and AMV 23102017 SBB 21920170589 according to institutional guidelines.

Syrian hamsters were purchased from Janvier Laboratories and were housed per two in ventilated isolator cages (IsoCage N Biocontainment System, Tecniplast) with ad libitum access to food and water and cage enrichment (wood block). Housing conditions and experimental procedures were approved by the ethical committee of animal experimentation of KU Leuven (licence P065-2020). Hamsters were randomized and the sample size was chosen on the basis of previous experience with this animal model. To evaluate the prophylactic efficacy 6-10-week-old female hamsters were administered by intraperitoneal injection with S2X259 mAb at 1, 4, 5 and $25 \mathrm{mg} \mathrm{kg}^{-1}$ or a combination of S2X259 and S309 mAbs $\left(1 \mathrm{mg} \mathrm{kg}^{-1}+1 \mathrm{mg} \mathrm{kg}^{-1}\right)$ $48 \mathrm{~h}$ before intranasal infection with $1.89 \times 10^{6} \mathrm{TCID}_{50}$ in $50 \mu \mathrm{l}$ inoculum. Blood samples were collected before infection and serum was obtained for pharmacokinetics analysis. To assess the therapeutic efficacy, 6-8-week-old Syrian hamsters were administered by intraperitoneally with isotype control antibody $\left(20 \mathrm{mg} \mathrm{kg}^{-1}\right)$ or S2X259 $\mathrm{mAb}$ (at 20,10 or $5 \mathrm{mg} \mathrm{kg}^{-1}$ ) $24 \mathrm{~h}$ after infection with SARS-CoV-2. Blood samples were collected when hamsters were killed, and serum was obtained for pharmacokinetics analysis. Hamsters were monitored for appearance, behaviour and weight. At day 4 after infection, hamsters were euthanized by intraperitoneal injection of $500 \mu$ l Dolethal (200 $\mathrm{mg} \mathrm{ml}^{-1}$ sodium pentobarbital, Vétoquinol SA). Lungs were collected, homogenized using bead disruption (Precellys) in $350 \mu \mathrm{l}$ RLT buffer (RNeasy Mini kit, Qiagen) and centrifuged (10,000 r.p.m., $5 \mathrm{~min}, 4^{\circ} \mathrm{C}$ ) to pellet the cell debris. RNA was extracted using a NucleoSpin kit (Macherey-Nagel) according to the manufacturer's instructions. RT-qPCR was performed on a LightCycler96 platform (Roche) using the iTaq Universal Probes One-Step RTqPCR kit (BioRad) with $\mathrm{N} 2$ primers and probes targeting the nucleocapsid ${ }^{36}$. Standards of SARS-CoV-2 cDNA (IDT) were used to express viral genome copies per $\mathrm{mg}$ tissue or per $\mathrm{ml}$ serum. To quantify infectious SARS-CoV-2 particles, end-point titrations were performed on confluent Vero E6 cells in 96-well plates. The hamster-derived samples for RNA quantification and viral load titration were run by technicians who were blinded to treatment group of the analysed samples. Viral titres were calculated by a previously published method $^{55}$ and were expressed as $\mathrm{TCID}_{50}$ per mg tissue.

\section{Evaluation of escape mutants using deep-mutational scanning}

A previously described deep-mutational scanning approach ${ }^{56}$ was used to identify RBD mutations that escape S2X259 binding. In brief, duplicate libraries containing virtually all possible amino acid changes compatible with ACE2 binding and RBD folding within the Wuhan-Hu-1 SARS-CoV-2 RBD sequence were expressed on the surface of yeast ${ }^{29,56}$. Libraries were labelled at $59 \mathrm{ng} \mathrm{ml}^{-1} \mathrm{~S} 2 \mathrm{X} 259$ antibody (the $90 \%$ effective concentration for binding to yeast-displayed SARS-CoV-2 RBD determined in isogenic pilot binding experiments), and fluorescence-activated cell sorting (FACS) was used to select RBD ${ }^{+}$ cells that exhibit reduced antibody binding, as previously described ${ }^{56,57}$. Libraries were sequenced before and after selection to determine per-mutation escape fractions, as previously described ${ }^{57}$. Experiments were performed in duplicate with independently generated mutant libraries, and we report the average mutant escape fraction across the duplicates. Raw escape fractions are available on GitHub: https://github. com/jbloomlab/SARS-CoV-2-RBD_MAP_Vir_mAbs/blob/main/results/ supp_data/s2X259_raw_data.csv. Detailed steps of analysis and code for the deep mutational scanning selections are available on GitHub: https://github.com/jbloomlab/SARS-CoV-2-RBD_MAP_Vir_mAbs.

\section{Selection of SARS-CoV-2 mAb escape mutants}

VSV-SARS-CoV-2 chimera was used to select for SARS-CoV-2 S mAb-resistant mutants, as previously described ${ }^{30,31}$. In brief, mAb-resistant mutants were recovered by plaque isolation on Vero cells with the indicated $\mathrm{mAb}$ in the overlay. The concentration of $\mathrm{mAb}$ in the overlay was determined by neutralization assays at a MOI of 100. Escape clones were plaque-purified on Vero cells in the presence of $\mathrm{mAb}$, and plaques in agarose plugs were amplified on MA104 cells with the mAb present in the medium. Viral stocks were amplified on MA104 cells at an MOI of 0.01 in Medium 199 containing $2 \% \mathrm{FBS}$ and $20 \mathrm{mM}$ HEPES pH 7.7 (Millipore Sigma) at $34^{\circ} \mathrm{C}$. Viral supernatants were collected upon extensive cytopathic effect and clarified of cell debris by centrifugation at $1,000 \mathrm{~g}$ for $5 \mathrm{~min}$. Aliquots were maintained at $-80^{\circ} \mathrm{C}$. Viral RNA was extracted from VSV-SARS-CoV-2 mutant viruses using RNeasy Mini kit (Qiagen), and S was amplified using OneStep RT-PCR Kit (Qiagen). The mutations were identified by Sanger sequencing (GENEWIZ). Their resistance was verified by subsequent virus infection in the presence or absence of antibody. In brief, Vero cells were seeded into 12-well plates for overnight. The virus was serially diluted using DMEM and cells were infected at $37^{\circ} \mathrm{C}$ for $1 \mathrm{~h}$. Cells were cultured with an agarose overlay in the presence or absence of $\mathrm{mAb}$ at $34^{\circ} \mathrm{C}$ for 2 days. Plates were scanned on a biomolecular imager and expression of eGFP is show at $48 \mathrm{~h}$ after infection.

\section{Crystallization, data collection, structure determination and analysis}

Crystals of the SARS-CoV-2-RBD-S2X259-S2H97 Fab complex were obtained at $20^{\circ} \mathrm{C}$ by sitting drop vapour diffusion. A total of $200 \mathrm{nl}$ complex at $5.7 \mathrm{mg} \mathrm{ml}^{-1}$ was mixed with $200 \mathrm{nl}$ mother liquor solution containing $0.12 \mathrm{M}$ monosaccharides mix, 20\% (v/v) ethylene glycol, 10\% (w/v) PEG 8000, 0.1 M Tris (base)/bicine pH 8.5, $0.02 \mathrm{M}$ sodium chloride, 0.01 M MES pH 6 and 3\% (v/v) Jeffamine ED-2003. Crystals were flash-frozen in liquid nitrogen. Data were collected at Beamline 9-2 of the Stanford Synchrotron Radiation Lightsource facility. Data were processed with the XDS software package ${ }^{58}$ for a final dataset of $2.65 \AA$ in space group P21. The RBD-S2X259-S2H97 Fab complex structure was solved by molecular replacement using phaser ${ }^{59}$ from a starting model consisting of SARS-CoV-2 RBD (PDB code 7JX3) and homology models of the S2X259 and S2H97 Fabs built using the Molecular Operating Environment (MOE) software package from the Chemical Computing Group (https://www.chemcomp.com). Several subsequent rounds of model building and refinement were performed using $\operatorname{Coot}^{60}$, ISOLDE $^{61}$, 
Refmac5 ${ }^{62}$ and MOE (https://www.chemcomp.com), to arrive at a final model for the ternary complex.

\section{Cryo-EM sample preparation, data collection and data processing}

Recombinantly expressed and purified Fab S2X259 and SARS-CoV-2S hexapro were incubated at $1 \mathrm{mg} \mathrm{ml}^{-1}$ with a 1.2 molar excess of Fab at $4^{\circ} \mathrm{C}$ during $1 \mathrm{~h}$. Three microlitres of the complex mixture were loaded onto freshly glow discharged R 2/2 UltrAuFoil grids (200 mesh) or lacey grids covered with a thin layer of manually added carbon, before plunge-freezing using a vitrobot MarkIV (ThermoFisher Scientific) with a blot force of 0 and 7-7.5 s blot time (for the UltrAuFoil grids) or with a blot force of -1 and $2.5 \mathrm{~s}$ blot time (for the lacey thin carbon grids) at $100 \%$ humidity and $21^{\circ} \mathrm{C}$.

Data were acquired on a FEI Titan Krios transmission electron microscope operated at $300 \mathrm{kV}$ and equipped with a Gatan K2 Summit direct detector and Gatan Quantum GIF energy filter, operated in zero-loss mode with a slit width of $20 \mathrm{eV}$. Automated data collection was carried out using Leginon ${ }^{63}$ at a nominal magnification of $130,000 \times$ with a super-resolution pixel size of $0.525 \AA$. The dose rate was adjusted to 8 counts per pixel per s, and each movie was fractionated in 50 frames of $200 \mathrm{~ms}$. Two datasets were collected from UltrAuFoil grids with the stage tilted $30^{\circ}$ and $55^{\circ}$ to circumvent particle preferential orientation. The third dataset was collected on lacey grids covered with a thin layer of carbon. The three datasets, with a total of 6,786 micrographs, were collected with a defocus range of between -0.8 and $-2 \mu \mathrm{m}$. For each dataset, movie frame alignment, estimation of the microscope contrast-transfer function parameters, particle picking and extraction were carried out using Warp ${ }^{64}$. Particle images were extracted with a box size of 800 pixels $^{2}$ and binned to 400 yielding a pixel size of $1.05 \AA$. The three datasets were merged and two rounds of reference-free $2 \mathrm{D}$ classification were performed using cryoSPARC ${ }^{65}$. Subsequently, one round of 3D classification with 50 iterations, using PDB code $6 \mathrm{VXX}$ as initial model, was carried out using Relion ${ }^{66,67}$ without imposing symmetry. Three-dimensional refinements were carried out using nonuniform refinement $^{68}$. Particle images from each dataset were subjected to Bayesian polishing ${ }^{69}$ using Relion before merging them to perform another round of nonuniform refinement in cryoSPARC followed by per-particle defocus refinement and again nonuniform refinement. To improve the density of the S-S2X259 interface, the particles were symmetry-expanded and subjected to a Relion focus 3D classification without refining angles and shifts using a soft mask encompassing the RBD and S2X259 variable domains. Particles belonging to classes with the best-resolved local density were selected and subjected to local refinement using cryoSPARC. Local resolution estimation, filtering and sharpening were carried out using CryoSPARC. Reported resolutions are based on the gold-standard Fourier shell correlation of 0.143 criterion and Fourier shell correlation curves were corrected for the effects of soft masking by high-resolution noise substitution ${ }^{70}$.

\section{Cryo-EM model building and analysis}

UCSF Chimera ${ }^{71}$ and $\operatorname{Coot}^{60}$ were used to fit atomic models (PDB code $6 \mathrm{VXX}$ or $6 \mathrm{VYB}$ ) into the cryo-EM maps and the Fab variable domains were manually built. S2E12 was built in the locally refined map and subsequently validated using the Fab crystal structure. Models were refined and relaxed using Rosetta using both sharpened and unsharpened maps ${ }^{72,73}$. Validation used Phenix ${ }^{74}$, Molprobity ${ }^{75}$ and Privateer ${ }^{76}$. Figures were generated using UCSF ChimeraX ${ }^{77}$.

\section{Material availability}

Materials generated in this study can be available on request and may require a material transfer agreement. The SARS-CoV-2 RBD mutant libraries (no. 1000000172) and unmutated parental plasmid (no.166782) are available on Addgene

\section{Reporting summary}

Further information on research design is available in the Nature Research Reporting Summary linked to this paper.

\section{Data availability}

All datasets generated and information presented in the study are available from the corresponding authors on reasonable request. The cryo-EM structure data and model are available from the PDB (and Electron Microscopy Data Bank) under accession codes 7RA8 (EMD-24347) for the global refinement of the SARS-CoV-2 S and S2X259 complex and 7RAL (EMD-24365) for the local refinement of the SARS-CoV-2S RBD and S2X259 Fab variable domains. The X-ray structure data and model has been deposited at PDB code 7M7W for RBD-S2H97-S2X259. Interactive escape maps and structural visualizations can be found at: https://jbloomlab.github.io/SARS-CoV-2-RBD_MAP_Vir_mAbs/.Raw Illumina sequencing data from deep mutational scanning experiments are available on NCBI SRA, BioSample SAMN18315604 (SARS-CoV-2 mutant selection data). A complete table of deep-mutational scanning antibody escape fractions is provided on GitHub: https://github. com/jbloomlab/SARS-CoV-2-RBD_MAP_Vir_mAbs/blob/main/results/ supp_data/all_antibodies_raw_data.csv.

\section{Code availability}

A repository containing all code, analysis and summary notebooks for the analysis of the SARS-CoV-2 deep-mutational scanning escape selections is available on GitHub at https://github.com/jbloomlab/ SARS-CoV-2-RBD_MAP_Vir_mAbs.

48. Pinto, D. et al. Structural basis for broad HIV-1 neutralization by the MPER-specific human broadly neutralizing antibody LNO1. Cell Host Microbe 26, 623-637 (2019).

49. Slater, G. S. C \& Birney, E. Automated generation of heuristics for biological sequence comparison. BMC Bioinformatics 6, 31 (2005).

50. Katoh, K. \& Standley, D. M. MAFFT multiple sequence alignment software version 7: improvements in performance and usability. Mol. Biol. Evol. 30, 772-780 (2013)

51. Ou, X. et al. Characterization of spike glycoprotein of SARS-CoV-2 on virus entry and its immune cross-reactivity with SARS-CoV. Nat. Commun. 11, 1620 (2020).

52. Crawford, K. H. D. et al. Protocol and reagents for pseudotyping lentiviral particles with SARS-CoV-2 spike protein for neutralization assays. Viruses 12, 513 (2020).

53. Takada, A. et al. A system for functional analysis of Ebola virus glycoprotein. Proc. Natl Acad. Sci. USA 94, 14764-14769 (1997).

54. Xie, X. et al. A nanoluciferase SARS-CoV-2 for rapid neutralization testing and screening of anti-infective drugs for COVID-19. Nat. Commun. 11, 5214 (2020).

55. Reed, L. J. \& Muench, H. A simple method of estimating fifty per cent endpoints. Am. J. Epidemiol. 27, 493-497 (1938).

56. Greaney, A. J. et al. Complete mapping of mutations to the SARS-CoV-2 spike receptor-binding domain that escape antibody recognition. Cell Host Microbe 29, 44-57 (2021).

57. Starr, T. N. et al. Prospective mapping of viral mutations that escape antibodies used to treat COVID-19. Science 371, 850-854 (2021).

58. Kabsch, W. Xds. Acta Crystallogr. D 66, 125-132 (2010).

59. McCoy, A. J. et al. Phaser crystallographic software. J. Appl. Crystallogr. 40, 658-674 (2007).

60. Emsley, P., Lohkamp, B., Scott, W. G. \& Cowtan, K. Features and development of Coot. Acta Crystallogr. D 66, 486-501 (2010).

61. Croll, T. I. ISOLDE: a physically realistic environment for model building into low-resolution electron-density maps. Acta Crystallogr. D 74, 519-530 (2018)

62. Murshudov, G. N. et al. REFMAC5 for the refinement of macromolecular crystal structures. Acta Crystallogr. D 67, 355-367 (2011).

63. Suloway, C. et al. Automated molecular microscopy: the new Leginon system. J. Struct. Biol. 151, 41-60 (2005).

64. Tegunov, D. \& Cramer, P. Real-time cryo-electron microscopy data preprocessing with Warp. Nat. Methods 16, 1146-1152 (2019).

65. Punjani, A., Rubinstein, J. L., Fleet, D. J. \& Brubaker, M. A. cryoSPARC: algorithms for rapid unsupervised cryo-EM structure determination. Nat. Methods 14, 290-296 (2017).

66. Zivanov, J. et al. New tools for automated high-resolution cryo-EM structure determination in RELION-3. eLife 7, e42166 (2018).

67. Kimanius, D., Forsberg, B. O., Scheres, S. H. \& Lindahl, E. Accelerated cryo-EM structure determination with parallelisation using GPUs in RELION-2. eLife 5, e18722 (2016).

68. Punjani, A., Zhang, H. \& Fleet, D. J. Non-uniform refinement: adaptive regularization improves single-particle cryo-EM reconstruction. Nat. Methods 17, 1214-1221 (2020)

69. Zivanov, J., Nakane, T. \& Scheres, S. H. W. A Bayesian approach to beam-induced motion correction in cryo-EM single-particle analysis. IUCrJ 6, 5-17 (2019). 


\section{Article}

70. Chen, S. et al. High-resolution noise substitution to measure overfitting and validate resolution in 3D structure determination by single particle electron cryomicroscopy. Ultramicroscopy 135, 24-35 (2013)

71. Pettersen, E. F. et al. UCSF Chimera-a visualization system for exploratory research and analysis. J. Comput. Chem. 25, 1605-1612 (2004).

72. Frenz, B. et al. Automatically fixing errors in glycoprotein structures with Rosetta. Structure 27, 134-139 (2019).

73. Wang, R. Y. et al. Automated structure refinement of macromolecular assemblies from cryo-EM maps using Rosetta. eLife 5, e17219 (2016).

74. Liebschner, D. et al. Macromolecular structure determination using X-rays, neutrons and electrons: recent developments in Phenix. Acta Crystallogr. D 75 861-877 (2019).

75. Chen, V. B. et al. MolProbity: all-atom structure validation for macromolecular crystallography. Acta Crystallogr. D 66, 12-21 (2010).

76. Agirre, J. et al. Privateer: software for the conformational validation of carbohydrate structures. Nat. Struct. Mol. Biol. 22, 833-834 (2015).

77. Goddard, T. D. et al. UCSF ChimeraX: meeting modern challenges in visualization and analysis. Protein Sci. 27, 14-25 (2018).

78. Pinto, D. et al. A human antibody that broadly neutralizes betacoronaviruses protects against SARS-CoV-2 by blocking the fusion machinery. Preprint at https://doi. org/10.1101/2021.05.09.442808 (2021).

Acknowledgements We thank $\mathrm{H}$. Tani for providing the reagents necessary for preparing VSV pseudotyped viruses; C. Castado and N. Blais for their help in the selection of the genetically divergent sarbecoviruses used in this study; J. C. Nix for X-ray data collection I. Hoffman and T. I. Croll for assistance in refinement of crystal structures; and J. Dillen for assistance with surface plasmon resonance data collection. This study was supported by the National Institute of General Medical Sciences (R01GM120553 to D.V.), the National Institute of Allergy and Infectious Diseases (DP1Al158186 and HHSN272201700059C to D.V.; R01Al141707 to J.D.B.), a Pew Biomedical Scholars Award (D.V.), Investigators in the Pathogenesis of Infectious Disease Awards from the Burroughs Wellcome Fund (D.V.), Fast Grants (DV), the Pasteur Institute (M.A.T), the Damon Runyon Cancer Research Foundation (T.N.S.) and the University of Washington Arnold and Mabel Beckman cryo-EM centre. J.D.B. is an Investigator of the Howard Hughes Medical Institute. Use of the Stanford Synchrotron Radiation Lightsource, SLAC National Accelerator Laboratory, is supported by the US Department of Energy, Office of Science, Office of Basic Energy Sciences under contract no. DE-AC02-76SF00515. The SSRL Structural Molecular Biology Program is supported by the DOE Office of Biological and Environmental Research, and by the National Institutes of Health, National Institute of General Medical Sciences (P30GM133894). The contents of this publication are solely the responsibility of the authors and do not necessarily represent the official views of NIGMS or NIH.
Author contributions Experiment design was performed by M.A.T., N.C., T.N.S., R.M., A.C.W., S.P.J.W., J.D.B., D.C., D.V. and M.S.P.; E.C. and F.B. recruited donors and collected samples; R.M F.Z., A.D.M., D.P. and M.B. processed PBMC samples; M.A.T., N.C., S.J., A.C.W., J.E.B., K.C., N.S., C.S., I.G.-S. and E.C. performed protein expression and purification; R.M., F.Z., A.D.M., D.P., M.B. and C.S.F. isolated mAbs; M.A.T., R.M., A.C.W., F.Z., D.P., M.B., M.P.H., F.A.L., E.D. Jr, H.K., M.M.-R., J.Z., S.K.Z. and C.H.-D. performed binding and neutralization assays; A.C.W., F.Z., A.D.M. and L.E.R. undertook biolayer interferometry and surface plasmon resonance assays; M.A.T., Z.W. and D.V. collected and processed cryo-EM data, and built the models; N.C. and G.S. performed crystallization experiments and determined the X-ray structure; D.P. and A.D.M. performed the ACE2 binding inhibition and S1 shedding; B.G. evaluated effector functions; T.N.S., A.A. and J.D.B. performed deep-mutational scanning; J.D.I., I.B. and A.T. undertook bioinformatic analysis of virus diversity and variants; Z.L. and S.P.J.W. selected and sequenced escape mutants; R.A., S.-Y.C.F., E.V., M.A.S., F.B., J.N., D.C. and M.S.P. performed hamster model experiments and data analysis; M.A.T., N.C., T.N.S., R.M., A.C.W., J.D.B., D.C., D.V. and M.S.P. analysed data; M.A.T., N.C., T.N.S., R.M., J.N., A.T., G.S., H.W.V., S.P.J.W., J.D.B., D.C., D.V. and M.S.P. wrote the manuscript; F.A.L., L.E.R., C.H.-D., M.A.S., F.B., E.C., J.N., A.T., G.S., H.W.V., S.P.J.W., J.D.B., D.C., D.V. and M.S.P. supervised the project; and J.D.B. and D.V. acquired funding.

Competing interests N.C., R.M., F.Z., S.J., J.D.I., A.D.M., D.P., M.B., I.B., M.H., F.A.L., L.E.R, E.D. Jr, H. K., M.M.-R., J.Z., K.C., N.S., C.S., E.V., I.G.-S., C.S.F., C.H.-D., M.A.S., F.B., E.C., A.T., G.S., H.W.V., D.C. and M.S.P. are employees of Vir Biotechnology Inc. and may hold shares in Vir Biotechnology Inc. D.C. is currently listed as an inventor on multiple patent applications, which disclose the subject matter described in this Article. The laboratory of J.N. has received sponsored research agreements from Vir Biotechnology Inc. After the submission of the initial version of this study, J.D.B. began consulting for Moderna on viral evolution and epidemiology. J.D.B. has the potential to receive a share of IP revenue as an inventor on a Fred Hutchinson Cancer Research Center-optioned technology and patent (application WO2020006494) related to deep-mutational scanning of viral proteins. H.W.V. is a founder of PierianDx and Casma Therapeutics. Neither company provided funding for this work or is performing related work. D.V. is a consultant for Vir Biotechnology Inc. The laboratory of D.V. has received a sponsored research agreement from Vir Biotechnology Inc. The remaining authors declare that the research was conducted in the absence of any commercial or financial relationships that could be construed as a potential conflict of interest.

Additional information

Supplementary information The online version contains supplementary material available at https://doi.org/10.1038/s41586-021-03817-4.

Correspondence and requests for materials should be addressed to D.C., D.V. or M.S.P.

Peer review information Nature thanks the anonymous reviewers for their contribution to the peer review of this work.

Reprints and permissions information is available at http://www.nature.com/reprints. 
a

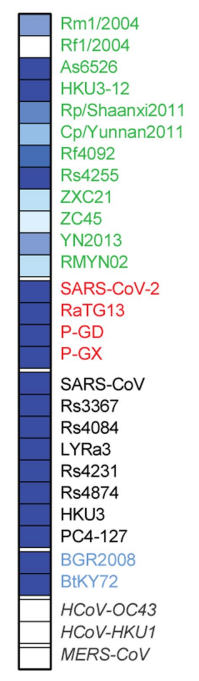

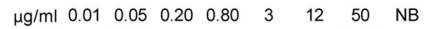

C

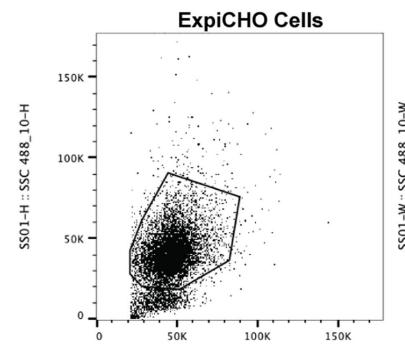

FS00-H :: FSC 488_10-H
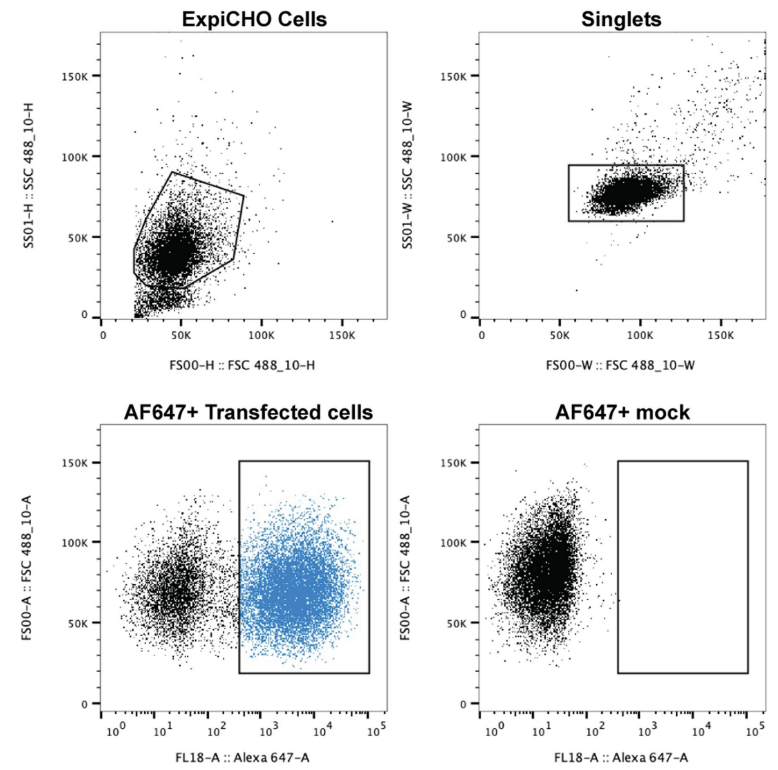

FS00-W : FSC 488_10-W

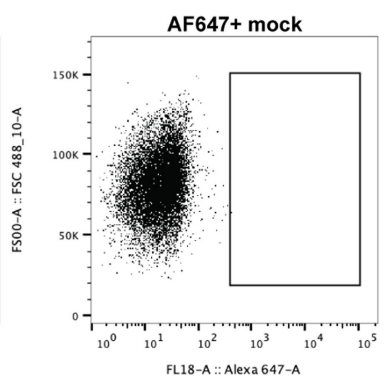

Extended Data Fig. 1 | Breadth of S2X259 mAb across the Sarbecovirus

subgenus. a, Flow cytometry analysis of S2X 259 cross-reactivity with a panel of $26 \mathrm{~S}$ glycoproteins representative of sarbecovirus clades $1 \mathrm{a}, 1 \mathrm{~b}, 2$ and 3 . The colours represent the lowest concentration of $\mathrm{mAb}$ at which binding was observed. b, FACS binding of S2X259 to a panel of sarbecovirus S glycoproteins transiently expressed in ExpiCHO cells. Results represent the binding b

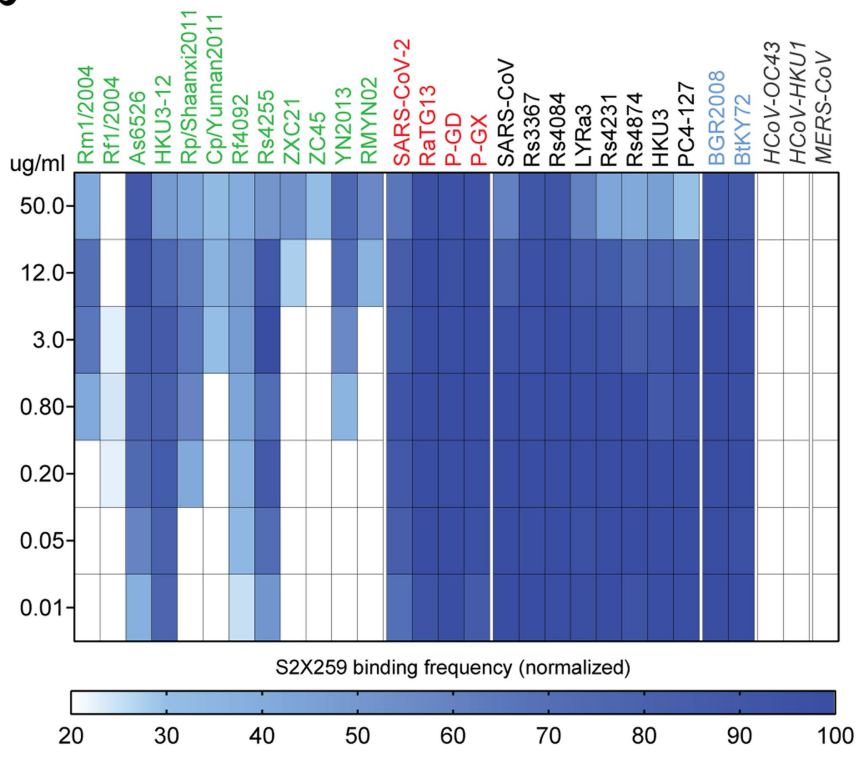

d

\begin{tabular}{|c|c|c|c|c|}
\hline & S2X259 & S2X35 & S2E12 & S309 \\
\hline SARS-CoV- & 9.97 & 25.58 & & 15.52 \\
\hline WIV1- & 11.04 & & & 17.06 \\
\hline SARS-CoV-2- & 31.96 & 28.20 & 5.82 & 10.92 \\
\hline RatG13- & 21.88 & 36.81 & 7.50 & 17.93 \\
\hline PangGD- & 28.45 & 33.00 & 6.91 & 15.88 \\
\hline Anlong112- & 1281.00 & & & \\
\hline YN2013- & 12.86 & 348.20 & & \\
\hline SC2018- & 11.63 & & & \\
\hline SX2011- & 26.50 & & & 239.80 \\
\hline zC45- & 539.00 & & & \\
\hline BtKY72- & 22.18 & 27.24 & & \\
\hline BGR2008- & 10.28 & 89.56 & & 22.09 \\
\hline $\begin{array}{l}\mathrm{EC5} 5 \\
\mathrm{ng} / \mathrm{ml}\end{array}$ & $1-50$ & $50-1000$ & $1000-5000$ & No binding \\
\hline
\end{tabular}

frequency normalized using the ratio between mean fluorescence intensities for S2X259 and an expression control $\mathrm{mAb}^{78}$.c, FACS gating strategy used to assess binding of S2X259 or of the expression control mAb.d, ELISA binding of site-I-targeting S2E12 $2^{21}$, site-II-targeting S2X 259 and S2X $35^{17}$, and site-IV-targeting $\mathrm{S} 309^{22} \mathrm{mAbs}$ to RBDs spanning the different clades of the Sarbecovirus subgenus. $\mathrm{EC}_{50}$ s are reported. $n=2$ independent experiments. 
a
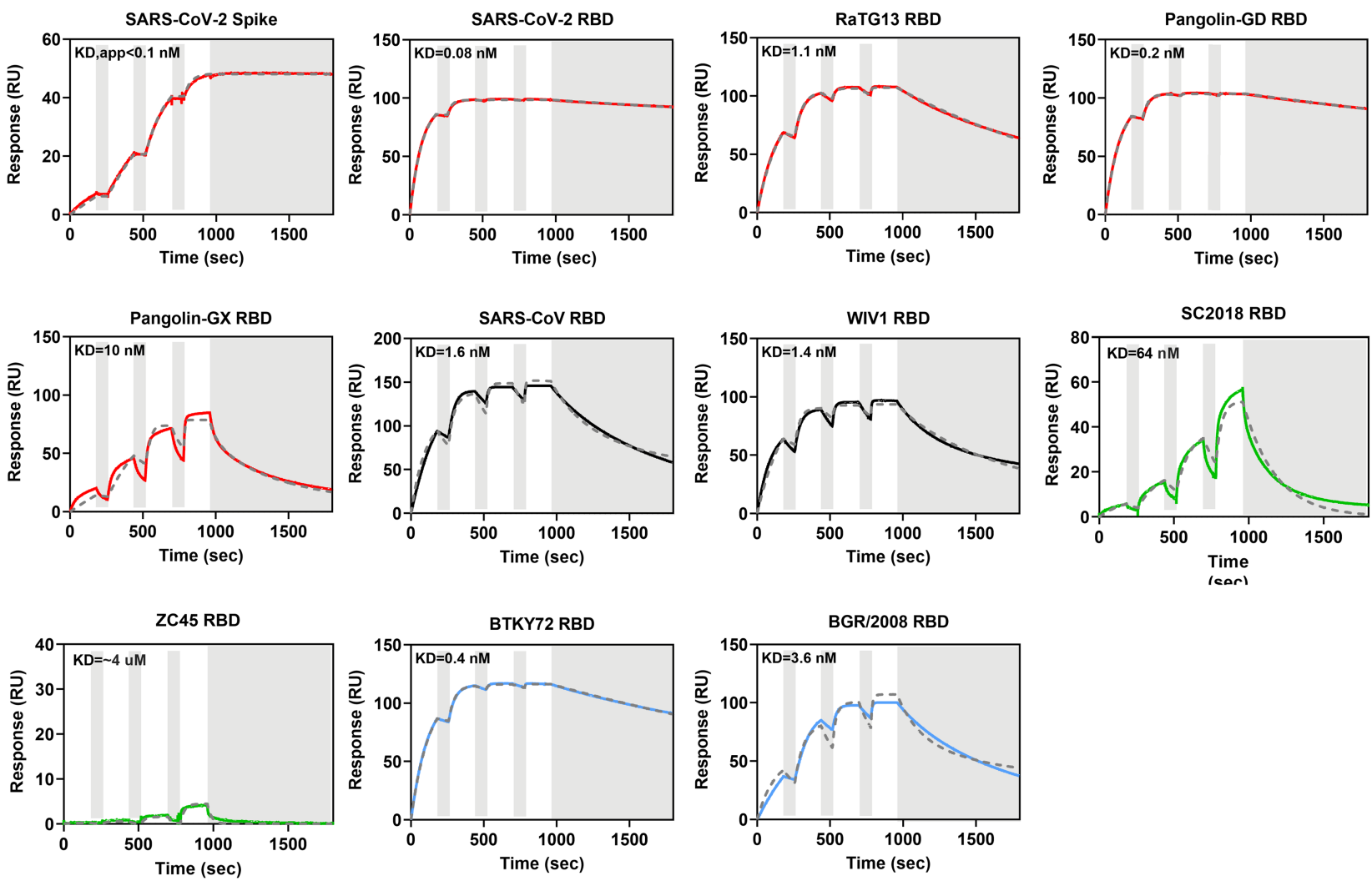

b

RBD WT

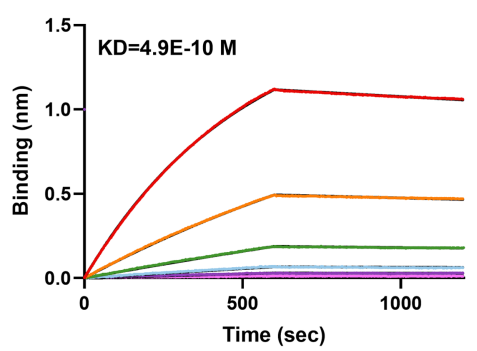

RBD P.1

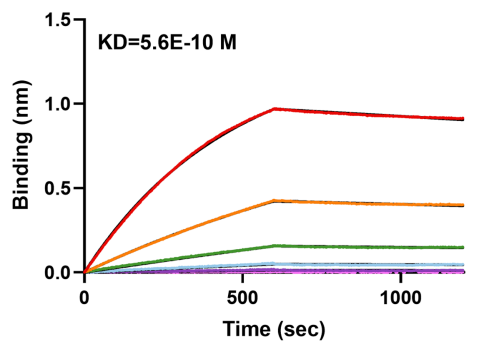

RBD B.1.1.7

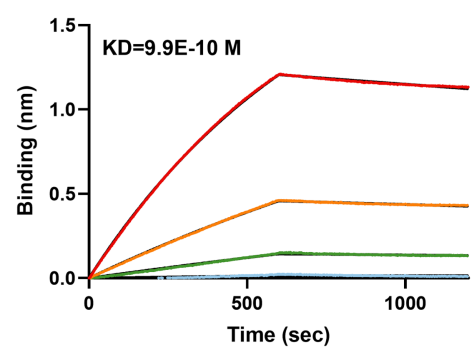

RBD B.1.429

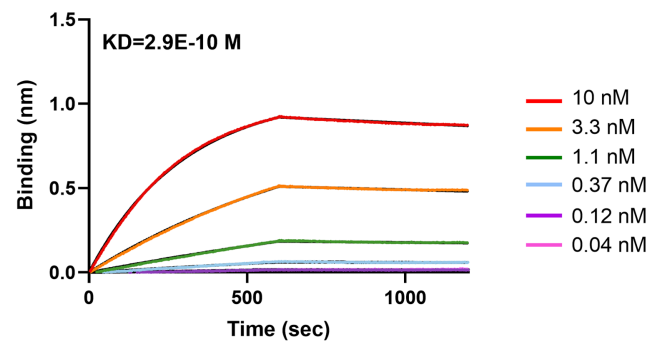

Extended Data Fig. 2 | S2X259 Fab binding to recombinant sarbecovirus RBDs, prefusion SARS-CoV-2 S ectodomain trimer and RBD variants. $a$, $S$ or RBD antigens were captured on the sensor chip surface and binding to $\$ 2 \times 259$ Fab at $11,33,100$ and $300 \mathrm{nM}$ was monitored successively, in single-cycle kinetics format, by surface plasmon resonance. All data have been fit to a 1:1 binding model (black dashed line) and the equilibrium dissociation constant $\left(K_{\mathrm{D}}\right)$ is reported. For the S-binding data, we report an apparent $K_{\mathrm{D}}\left(K_{\mathrm{D}, \text { app }}\right)$ as kinetics are affected by conformational dynamics between open and closed RBD states. The colouring scheme matches the phylogenetic tree in Fig. 1a. b, Biolayer interferometry binding analysis of the S2X259 Fab to wild-type or variant-of-concern SARS-CoV-2 biotinylated RBDs immobilized at the surface of SA biosensors. The data are coloured according to the key and fits to a 1:1 binding model are shown as black lines. Equilibrium dissociation constants $\left(K_{\mathrm{D}}\right)$ are reported above each plot.
RBD B.1.351

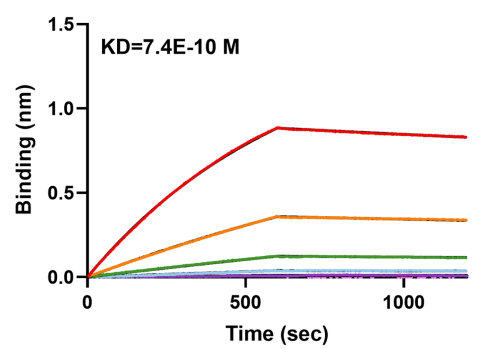


a

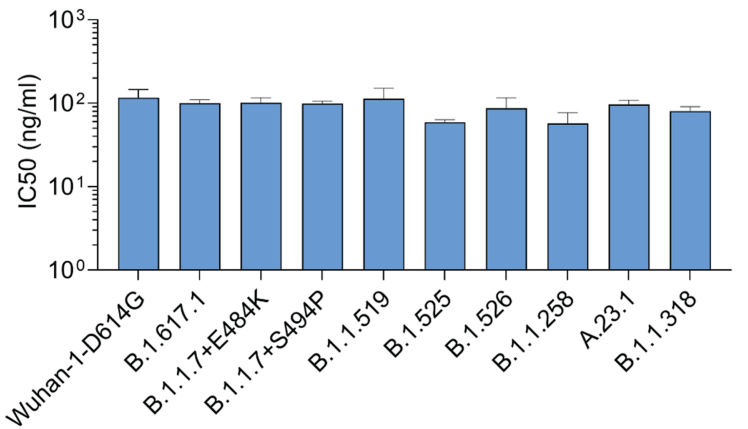

b

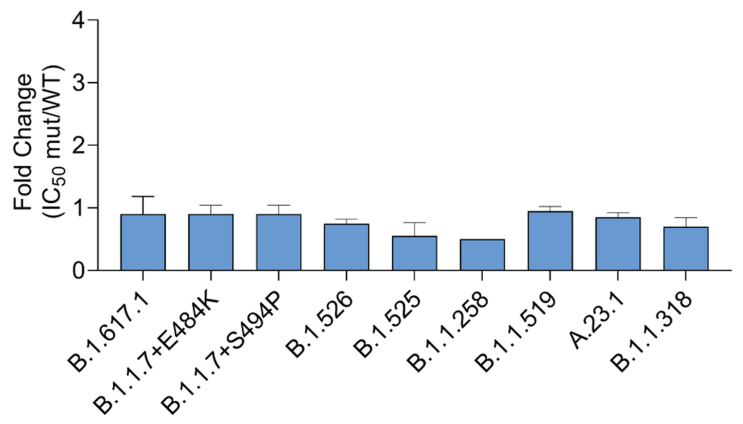

B.1.1.7 (UK)

NTD

RBD

$\mathrm{S} 1 / \mathrm{S} 2$

S2

\begin{tabular}{llll}
\hline 69-70del/144-del & N501Y/I(E484K or S494P) A570D/D614G/P681H T7161/S982A/D1118H \\
\hline
\end{tabular}

B.1.351 (South Africa)

P.1 (Brazil) L18F/D80A/D215G/R2461/2242-244de

K417N/E484K/N501Y

A701V

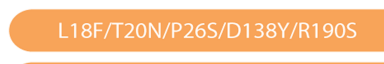

K417T/E484K/N501Y

T1027IN1167F

B.1.427/B.1.429 (California)

B.1.1.258 (Scotland)

B.1.525 (Nigeria)

B.1.526 (New York)

A.23.1(Liverpool)

B.1.1.318

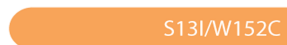

L452R

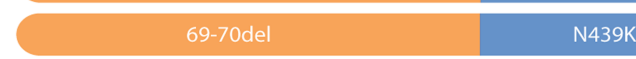

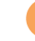

$$
\text { Q52R/A67V/69-70del/144del }
$$

E484K

F888L

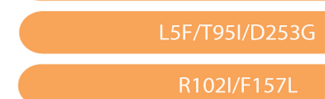

E484K

A701V

B.1.298 (Denmark)*

B.1.617.1 (India)

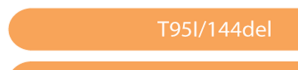

V367F/E484K

D796H

B.1.1.519 (Mexico/US)

\begin{tabular}{|c|c|c|c|}
\hline 69-70del & Y453F & D614G & \\
\hline E154K/T(T951/G142D & L452R/E484Q & D614G/P681R & Q1071H or H1101D \\
\hline & T478K & D614G/P681H & T732A \\
\hline
\end{tabular}

Extended Data Fig. 3 | S2X259 neutralizing activity is not affected by mutations present in circulating SARS-CoV-2 variant of concerns and recent lineages. $\mathbf{a}, \mathbf{b}$, Neutralization by $\mathrm{S} 2 \mathrm{X} 259$ of SARS-CoV-2 S VSV pseudotyped circulating variants shown as $\mathrm{IC}_{50}(\mathbf{a})$ and $\mathrm{IC}_{50}$ fold change as compared to Wuhan-Hu-1 virus with D614G substitution (b). Schematic of the SARS-CoV-2S and the mutation landscape in each variant is shown beneath the panels. Del, deletion. Asterisks denotes virus that was tested as single RBD mutant in Fig. 1e. 


\section{Article}

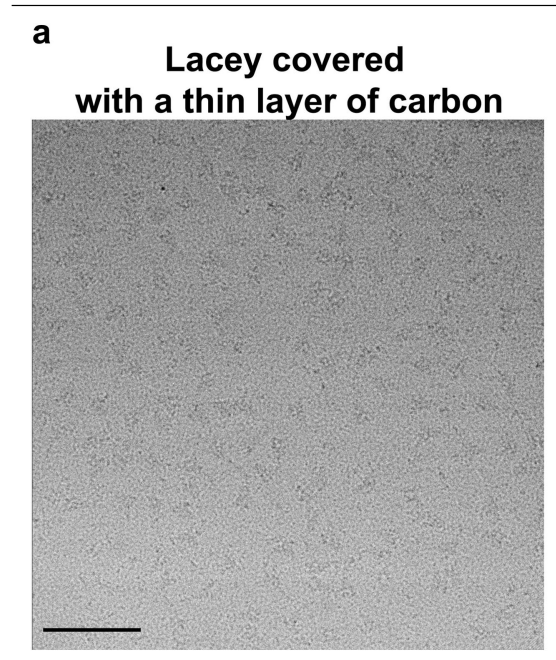

b
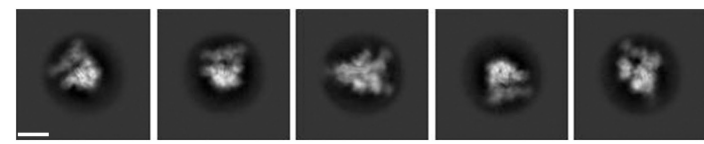

C

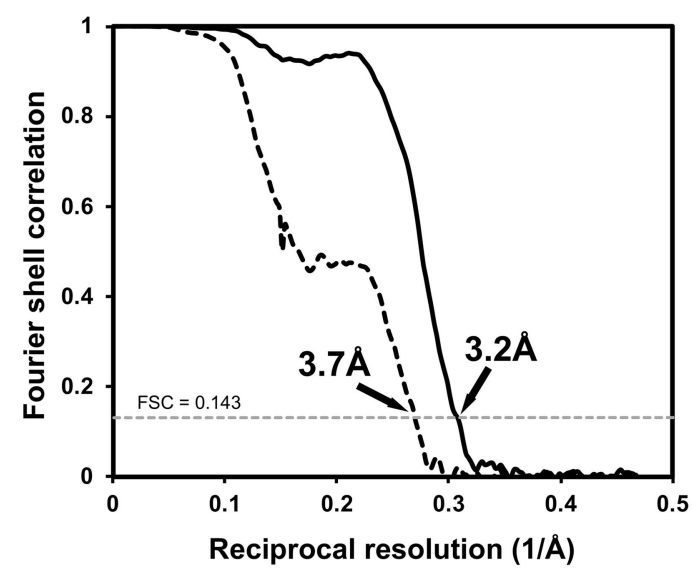

Extended Data Fig. 4 | Cryo-EM data processing and validation of S2X259-bound SARS-CoV-2S. a, b, Representative electron micrographs (a) and class averages (b) of SARS-CoV-2S in complex with the S2X259 Fab. Scale bars, $500 \AA$ (a), $100 \AA$ (b). c, Gold-standard Fourier shell correlation curves for the S trimer bound to three S2X259 Fabs (solid black line) and the locally d

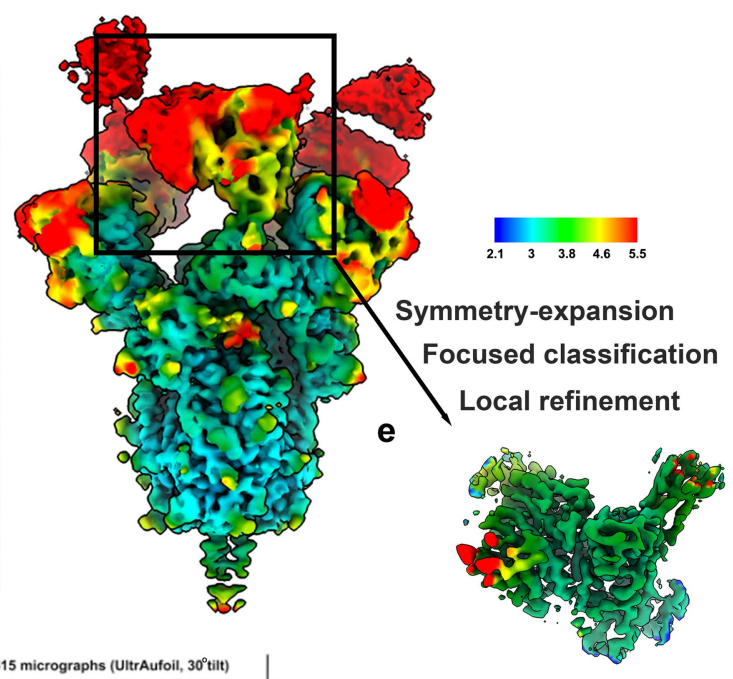

f

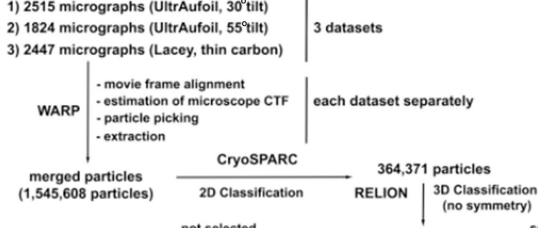

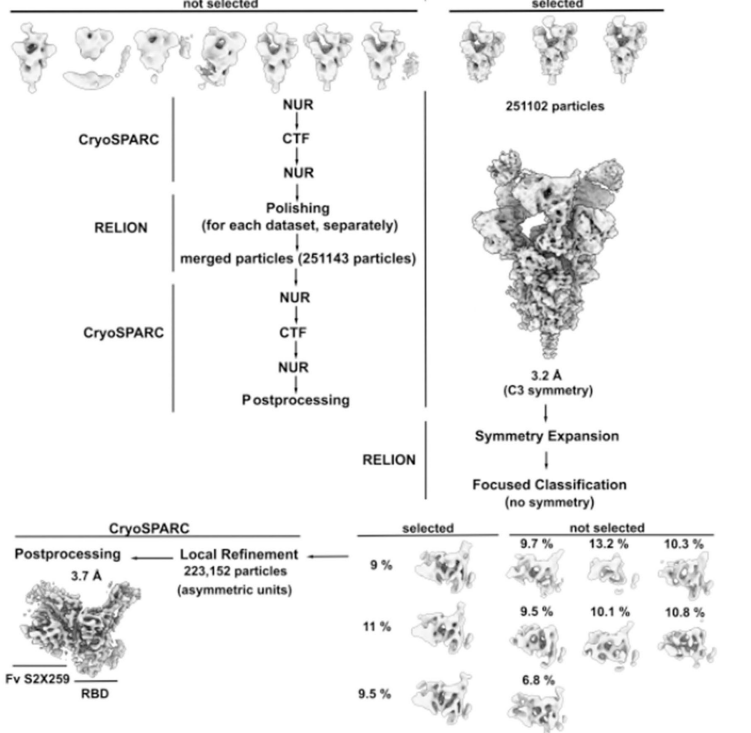

refined reconstruction of the RBD-S2X259 variable domains (dashed black line). The 0.143 cut-off is indicated by a horizontal dashed grey line. $\mathbf{d}, \mathbf{e}$, Local resolution map for the open S trimer bound to three S2X259 Fabs (d) and the locally refined reconstruction of the RBD-S2X259 variable domains (e). f, Cryo-EM data processing flowchart. 


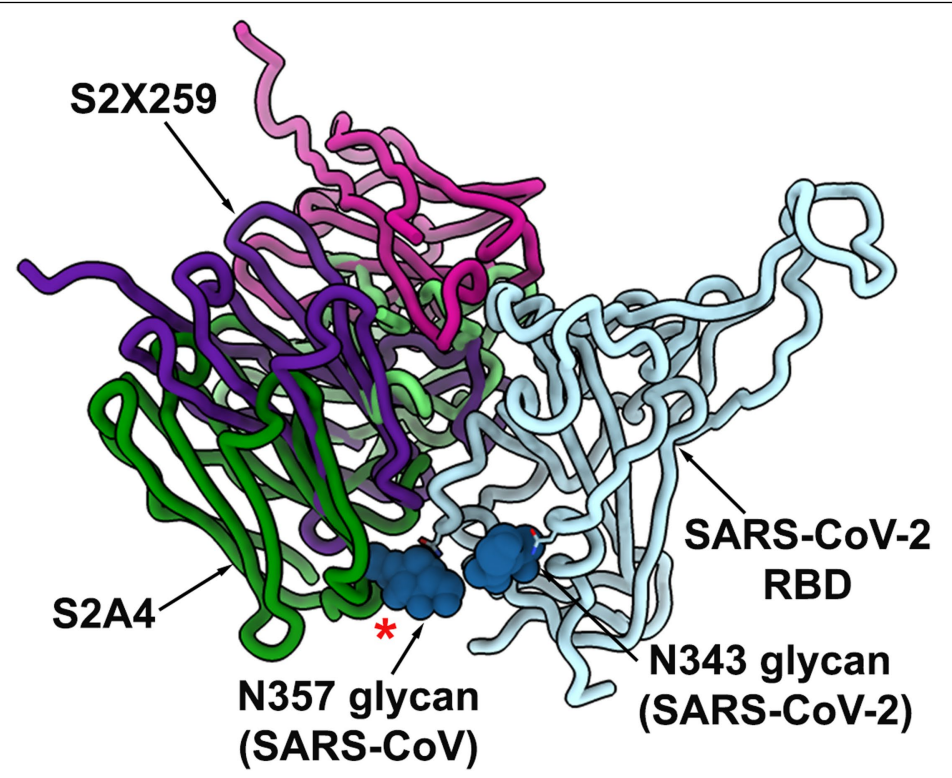

Extended Data Fig. 5 | The S2X259 angle of approach for binding to the SARS-CoV-2 RBD allows S2X259 to circumvent the SARS-CoV N357 glycan present in all sarbecovirus RBDs except SARS-CoV-2. Ribbon diagram showing a superimposition of the S2X259-bound and S2A4-bound (PDB code 7JVA) SARS-CoV-2 $\mathrm{RBD}^{17}$. The SARS-CoV glycan at position N357 was modelled

on the basis of the S230-bound SARS-CoV S structure (PDB code $6 \mathrm{NB}^{33}$ ) and is predicted to sterically hinder S2A4 binding (red star) but not S2X259 binding. The mAb light and heavy chains are coloured magenta and purple (S2X259) or light and dark green (S2A4), respectively. $\mathrm{N}$-linked glycans are rendered as blue spheres. 


\section{Article}

a

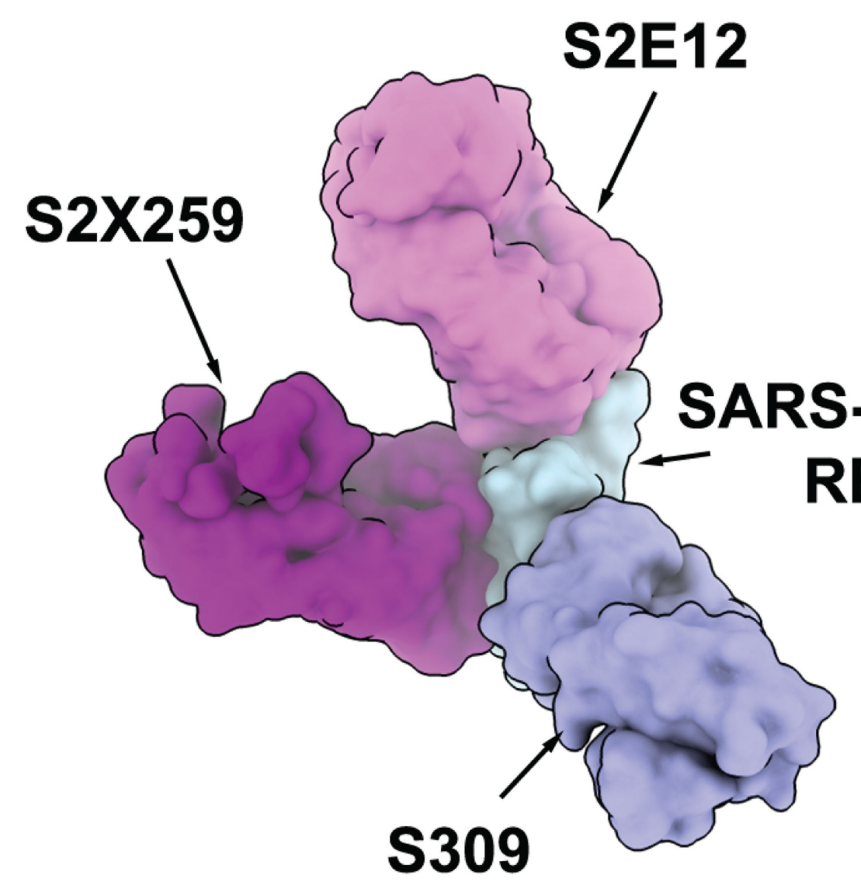

b

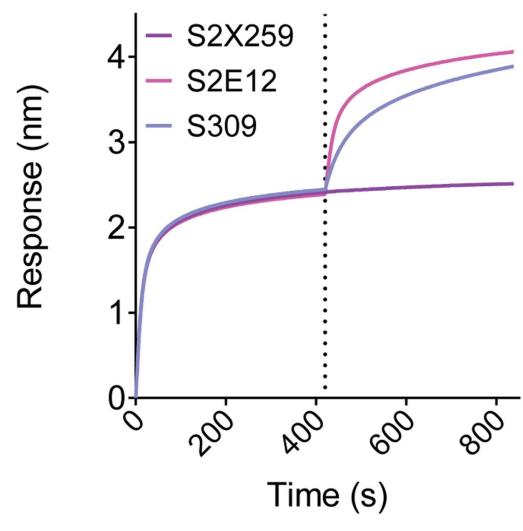

Extended Data Fig. 6 | S2X259 can bind to the SARS-CoV-2 RBD in presence of site-I and-IV-targeting $\mathbf{m A b s}$. a, View of site-I-targeting S2E12 ${ }^{21}$ (pink), site-II-targeting S2X259 (magenta) and site-IV-targeting S30 $9^{22}$ (purple) mAb bound to the SARS-CoV-2 RBD (light blue). b, Competition binding assays for S2X259 versus site-I-targeting S2E12 and site-IV-targeting S309 mAbs on SARS-CoV-2 RBD as measured by biolayer interferometry. One independent experiment out of two is shown.c, Competition ELISA (blockade-of-binding) between site-I-targeting S2H14 ${ }^{17}$ or site-II-targeting S2X259 and sera or plasma
Site I

- Site II

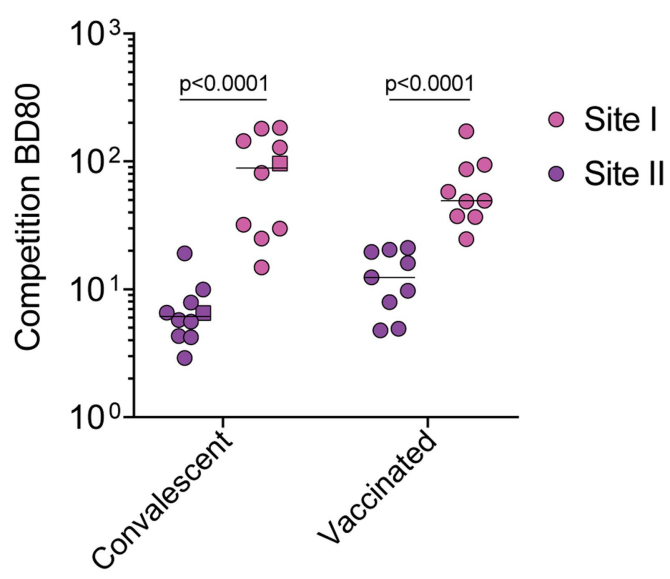

from individuals who had recovered from COVID-19 ( $n=10$ biological samples) and vaccinated individuals ( $n=9$ biological samples). Each plot shows the magnitude of inhibition of binding to immobilized RBD in the presence of each $\mathrm{mAb}$, expressed as reciprocal sera or plasma dilution blocking $80 \%$ of the maximum binding response. The individual from whom $\mathrm{S} 2 \mathrm{X} 259 \mathrm{mAb}$ was isolated is shown as a square. Statistical analysis was performed using two-tailed Mann-Whitney test. 
a
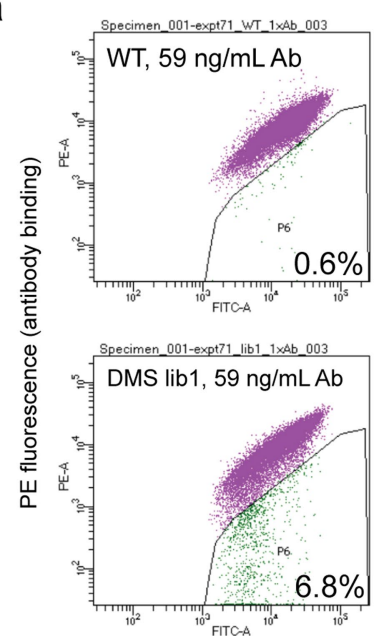

C
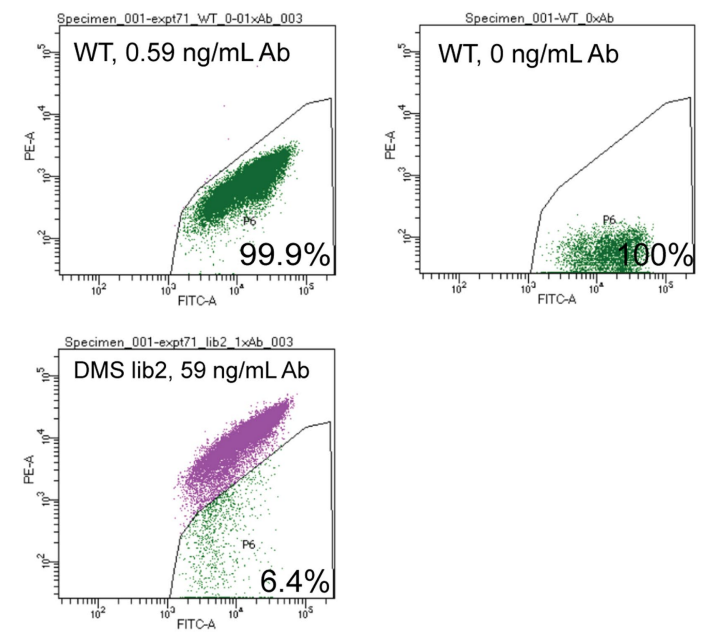

FITC fluorescence (RBD expression) b
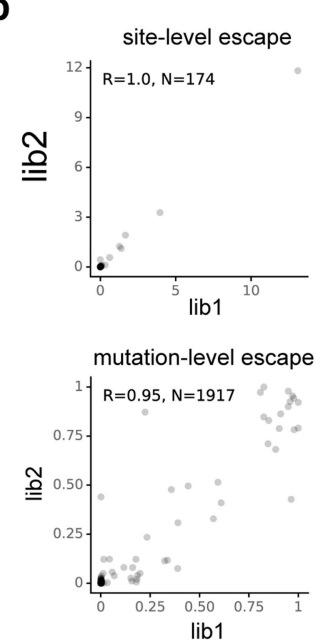

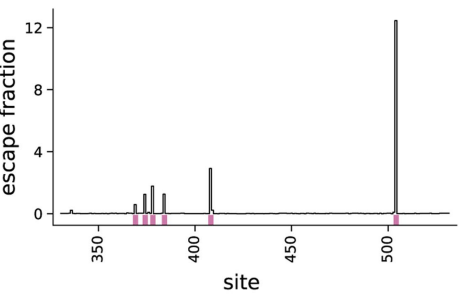

d

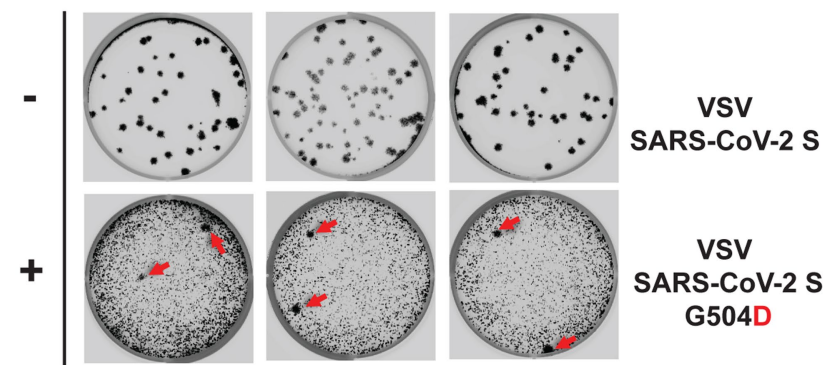

f

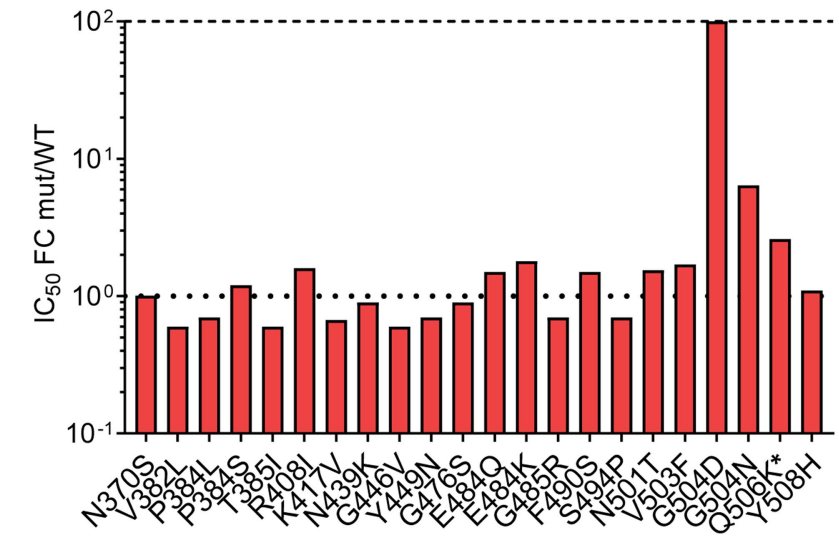

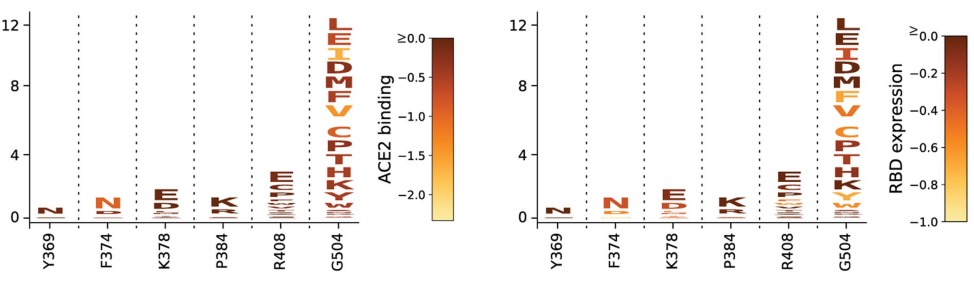

e

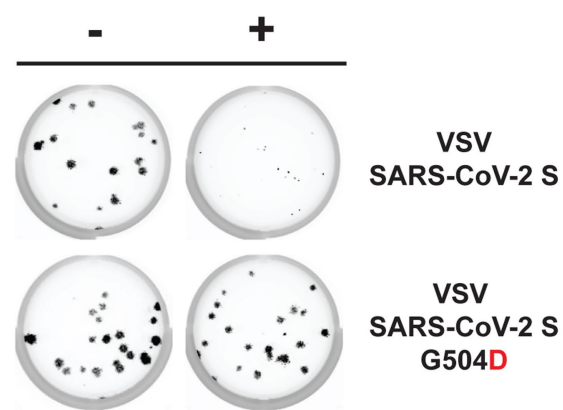

g

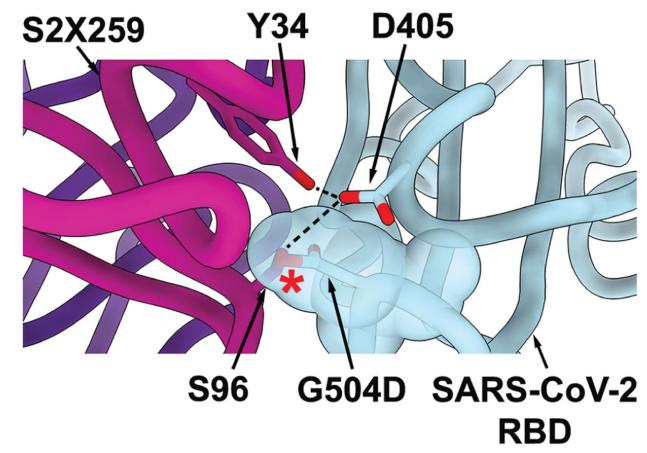

Extended Data Fig. 7 |See next page for caption. 


\section{Article}

Extended Data Fig. 7|S2X259 has a high barrier for the emergence of

resistance mutants. a, FACS gates used in deep-mutational scanning to select escape variants. Top, yeast controls expressing unmutated SARS-CoV-2 RBD labelled at relevant $\mathrm{S} 2 \mathrm{X} 259$ concentrations for setting of selection gates. Bottom, fraction of cells in SARS-CoV-2 mutant libraries falling into the antibody-escape bin.b, Correlation in site-level (top, sum of escape fractions for mutations at a site) and mutation-level (bottom) escape between independently generated and assayed RBD mutant libraries. c, Line plot of escape mutants along all positions in the SARS-CoV-2 RBD (left). Pink lines indicate sites that escape S2X259 binding illustrated at the mutation-level in logoplots (right). In logoplots, the height of a letter scales with its escape fraction. Letters are coloured according to their deleterious consequences for ACE2 binding (middle) or RBD expression (right) as determined in previous deep-mutational scanning experiments ${ }^{29}$.d, Plaque assay using VSV-SARSCoV-2 chimeric virus on Vero cells with no mAb (-) or S2X259 (+) in the overlay to isolate escape mutants (red arrow). Data are representative of three independent experiments. e, Plaque assays performed to validate the VSVSARS-CoV-2 G504D mutant in Vero cells in the presence $(+)$ or absence $(-)$ of S2X259 in the overlay. Representative image of two independent experiments is shown.f, S2X259 in vitro neutralizing activity against SARS-CoV-2S VSV pseudotyped mutants. For each mutant the fold change of the $\mathrm{IC}_{50}$ geometric mean versus SARS-CoV-2 S D614G is reported. ${ }^{*} \mathrm{Q} 506 \mathrm{~K}$ displayed a tenfold reduction in viral entry in comparison to the other mutants. Results from two independent experiments are reported.g, Zoomed-in view of the S2X259-RBD interface showing that the G504D substitution would disrupt $m A b$ binding owing to steric hindrance (indicated with a red star). 


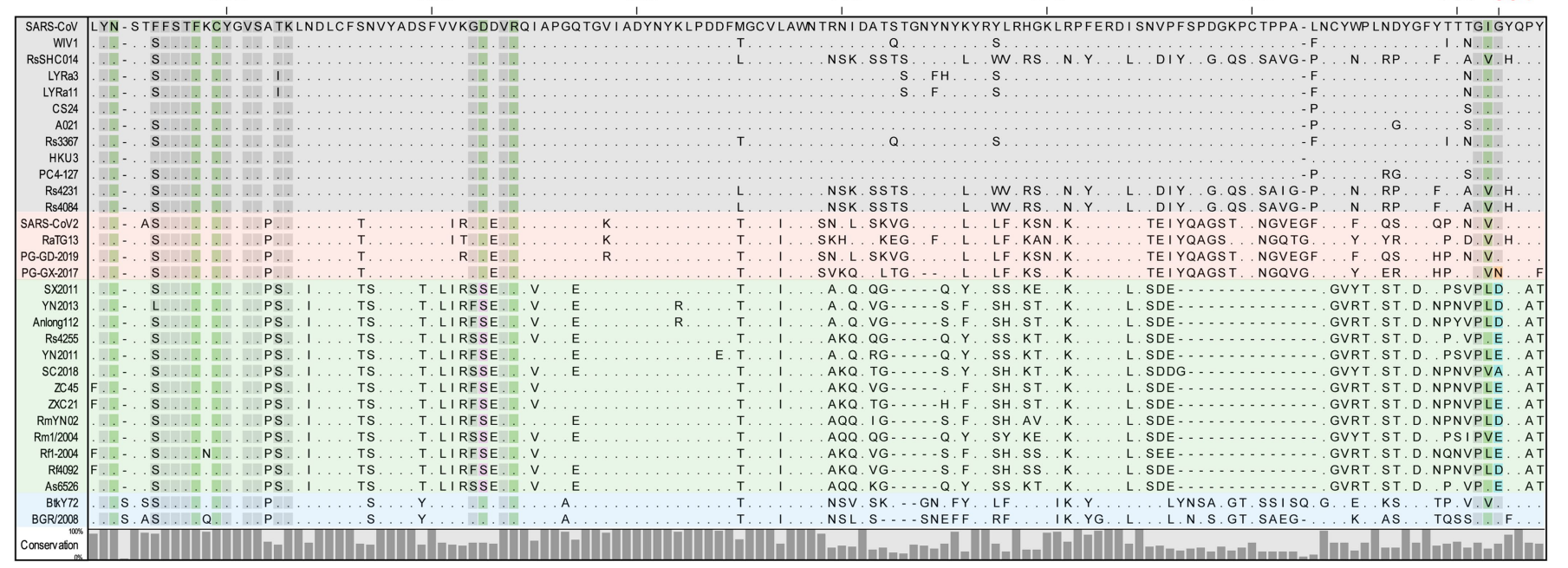

Extended Data Fig. 8 | S2X259 epitope conservation across sarbecovirus clades. Protein sequence alignment of representative sarbecovirus RBDs with strictly conserved residues shown as dots. Overall conservation is represented as a bar plot at the bottom of the figure. Residue positions are based on SARS-CoV-2. Residues determined to be most important for S2X259 binding are denoted in dark green. Substitutions at positions D405 and G504 are indicated in pink and blue or orange, respectively. Additional residues representing the extended epitope are denoted in grey. Different clades within the Sarbecovirus subgenus are overlaid in grey (clade 1a), red (clade 1b), green (clade 2) and light blue (clade 3 ). 
a

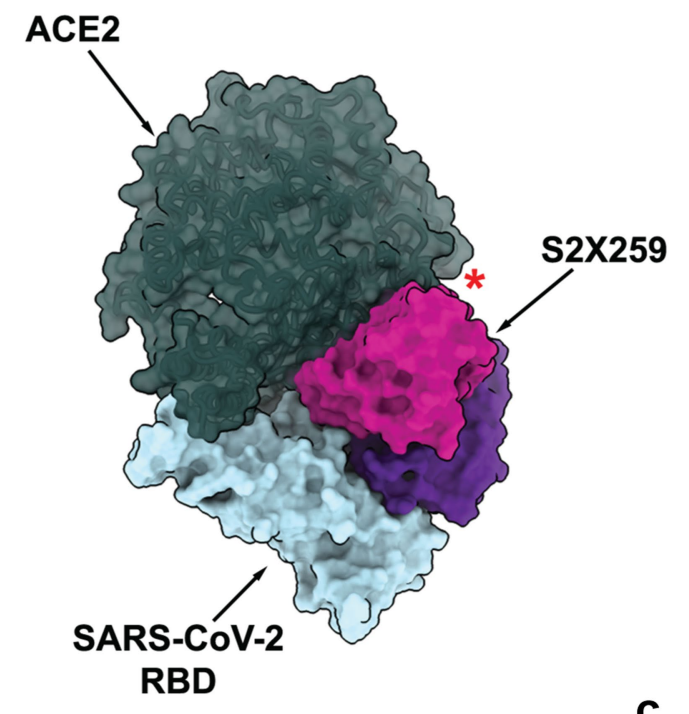

b

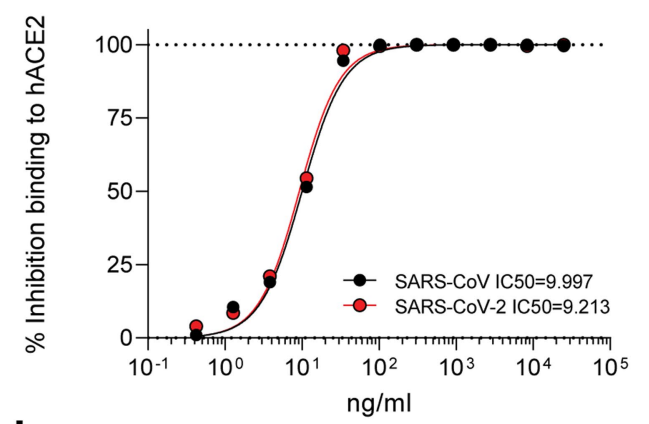

d

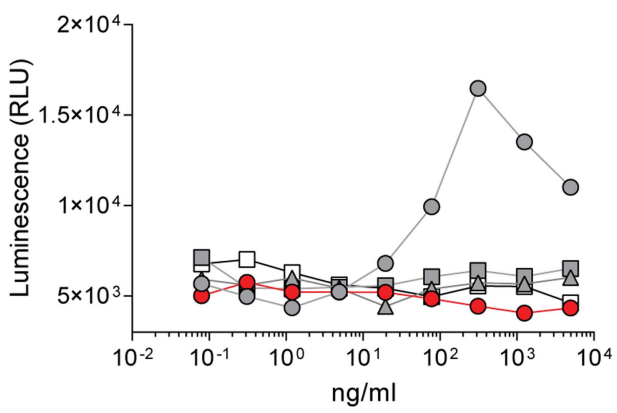

f

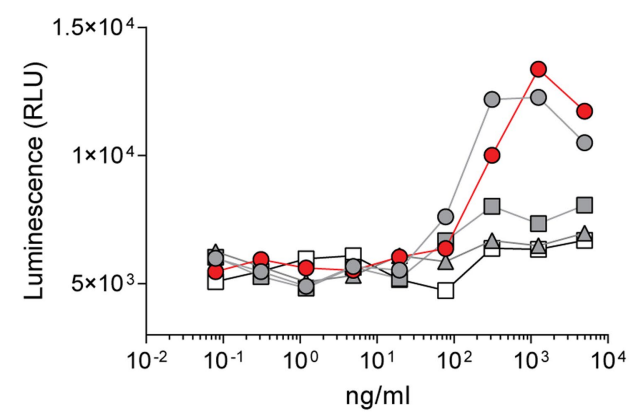

C

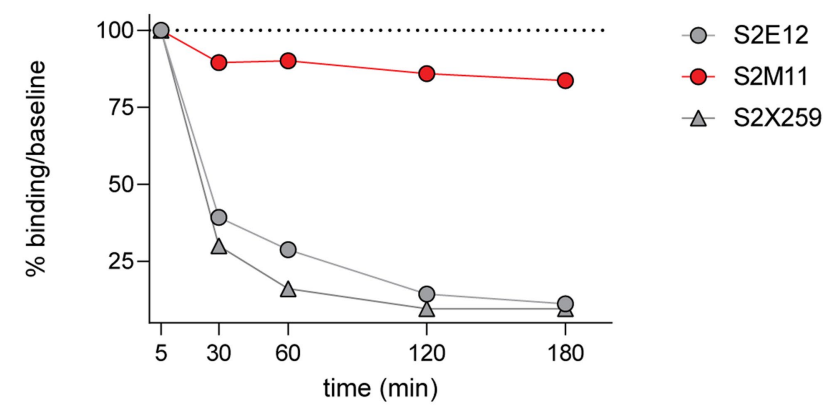

e

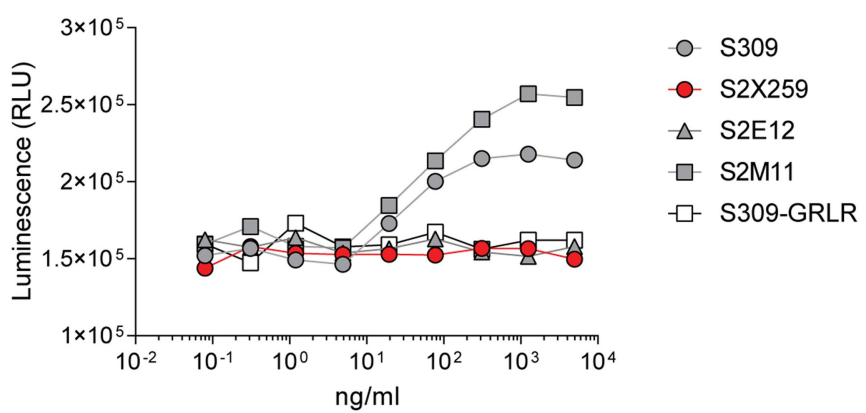

g

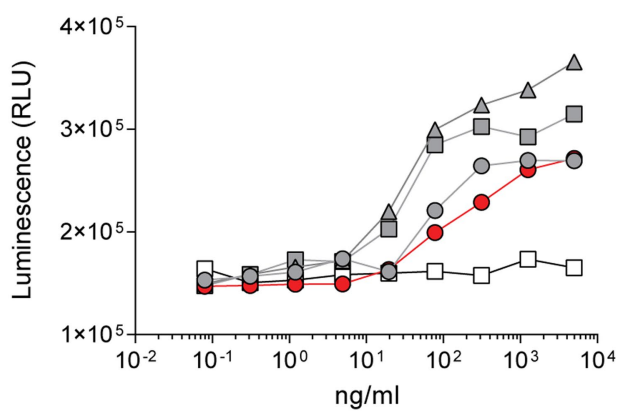

o- $\$ 309$

- $\mathrm{S} 2 \times 259$

$\triangle$ S2E12

ㄴ S2M11

마 S309-GRLR

Extended Data Fig. $9 \mid$ Inhibition of ACE2 engagement, S1 subunit shedding and activation of FcyRIIa and FcyRIIla in vitro. a, S2X259 (purple and pink) and ACE2 (dark green) bind partially overlapping binding sites on the SARS-CoV-2 RBD (blue). b, Pre-incubation of serial dilutions of S2X259 with SARS-CoV-2 (red) or the SARS-CoV (black) RBDs prevents binding to immobilized human ACE2 (hACE2) ectodomain in ELISA.c, mAb-mediated S1 subunit shedding from cell-surface-expressed SARS-CoV-2S as determined by flow cytometry. S2E12 was included as positive control and S2M11 was included

as negative control.d, e, NFAT-driven luciferase signal induced in Jurkat cells stably expressing FcүRIIa H131 (d) variant or FcүRIIIa V158 (e) variant by S2X259 binding to full-length wild-type SARS-CoV-2S on ExpiCHO target cells. $\mathbf{f}, \mathbf{g}$, NFAT-driven luciferase signal induced in Jurkat cells stably expressing Fc $ү$ RIIa H131 (f) or FcyRIIIa V158 (g) variants by S2X259 binding to uncleavable full-length prefusion stabilized SARS-CoV-2S (unable to release the $\mathrm{S} 1$ subunit) transiently expressed in ExpiCHO cells. SE12, S2M11, S309 and S309-GRLR mAbs are included as controls. 
a

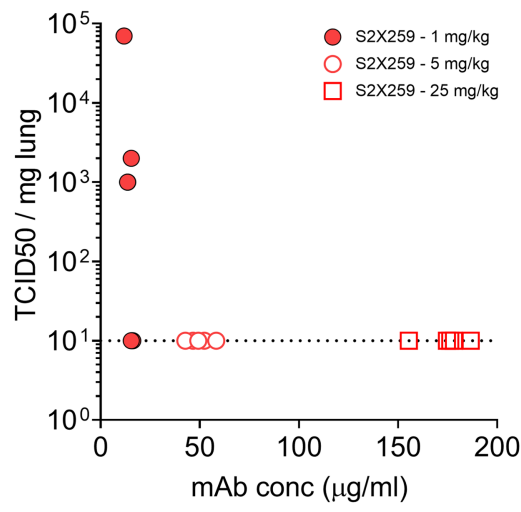

d

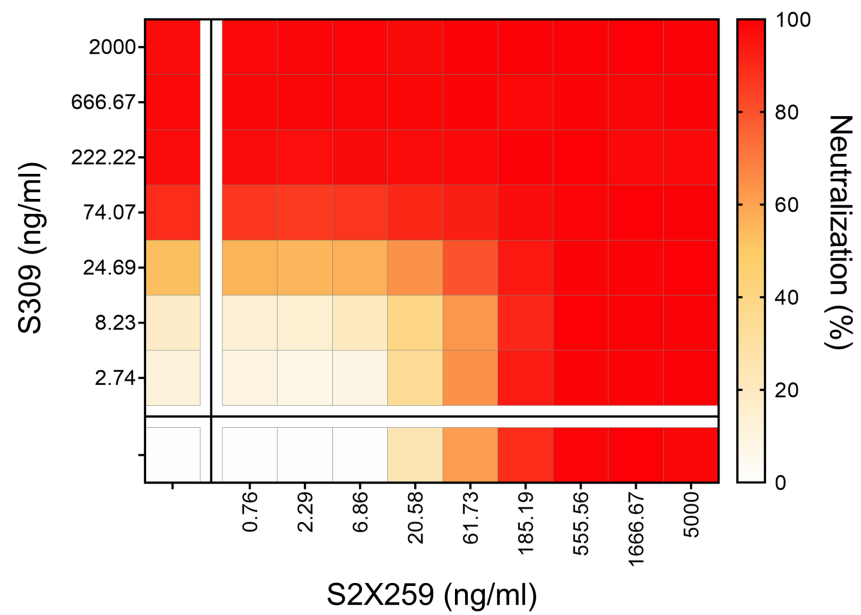

Extended Data Fig. 10 | Correlation between $\mathrm{mAb}$ concentration and infectious virus in vivo and in vitro neutralizing activity of

S2X259 + \$309 antibody cocktail. a-c, Infectious virus titres in the lungs at 4 days after infection plotted as a function of serum $m A b$ concentrations before infection (day 0) with prototypic SARS-CoV-2 (a) and SARS-CoV-2

B.1.351 (c) in prophylactic setting or at 4 days after infection with prototypic
C

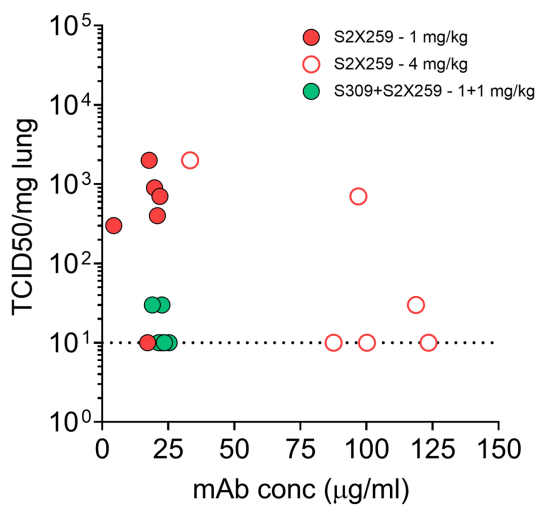

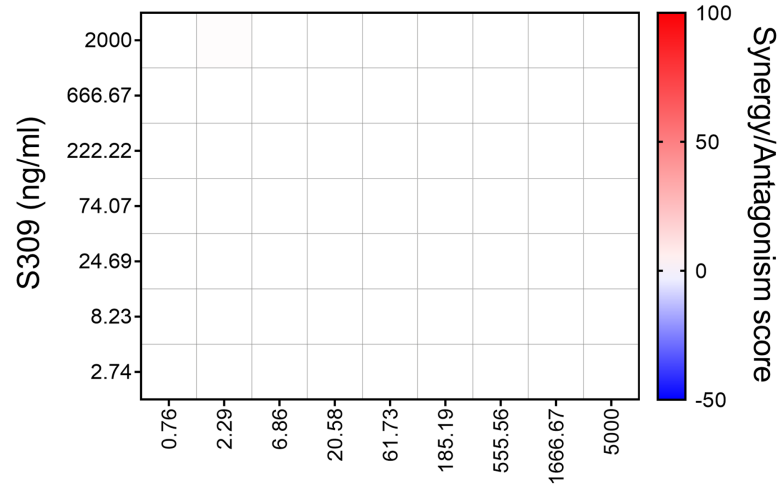

$\mathrm{S} 2 \times 259(\mathrm{ng} / \mathrm{ml})$ pseudotype neutralization (d) and synergy score (e) measured combining S2X259 and S309 mAbs. Synergy was calculated using MacSynergylI (synergy score: 0.98 , antagonism score: 0 ). Results represent four replicates of one independent experiment.

SARS-CoV-2 (b) in therapeutic setting. d, e, SARS-CoV-2 B1.351 VSV-based 


\section{Reporting Summary}

Nature Research wishes to improve the reproducibility of the work that we publish. This form provides structure for consistency and transparency in reporting. For further information on Nature Research policies, see our Editorial Policies and the Editorial Policy Checklist.

\section{Statistics}

For all statistical analyses, confirm that the following items are present in the figure legend, table legend, main text, or Methods section.

n/a Confirmed

$\square$ The exact sample size $(n)$ for each experimental group/condition, given as a discrete number and unit of measurement

$\square$ \ A statement on whether measurements were taken from distinct samples or whether the same sample was measured repeatedly

$\square$ The statistical test(s) used AND whether they are one- or two-sided

$\square$ Only common tests should be described solely by name; describe more complex techniques in the Methods section.

Х $\square$ A description of all covariates tested

$\bigotimes \square$ A description of any assumptions or corrections, such as tests of normality and adjustment for multiple comparisons

$\checkmark$ A full description of the statistical parameters including central tendency (e.g. means) or other basic estimates (e.g. regression coefficient)

$\bigotimes$ AND variation (e.g. standard deviation) or associated estimates of uncertainty (e.g. confidence intervals)

$\varnothing$ For null hypothesis testing, the test statistic (e.g. $F, t, r$ ) with confidence intervals, effect sizes, degrees of freedom and $P$ value noted Give $P$ values as exact values whenever suitable.

Х $\square$ For Bayesian analysis, information on the choice of priors and Markov chain Monte Carlo settings

Х $\square$ For hierarchical and complex designs, identification of the appropriate level for tests and full reporting of outcomes

Х $\square$ Estimates of effect sizes (e.g. Cohen's $d$, Pearson's $r$ ), indicating how they were calculated

Our web collection on statistics for biologists contains articles on many of the points above.

\section{Software and code}

Policy information about availability of computer code

Data collection As described in Methods:

* protein purification monitored by UNICORN software version 5.11

* SPR binding assays collected and analyzed using Biacore Evaluation and Biacore Insight software

* FACSDiva (version 6.1.3) and Everest 3.0 software for FACS data collection and cell sorting

* Identification of escape mutants in virological assay was performed by Sanger sequencing (GENEWIZ)

Data analysis Repository containing all code, analysis, and summary notebooks for the analysis of the SARS-CoV-2 deep mutational scanning escape selections available on GitHub: https://github.com/jbloomlab/SARS-CoV-2-RBD_MAP_Vir_mAbs. This code uses packages and versions as described in the Methods for various steps of computation.

Binding, Neutralization assays analyzed using PRISM (Version 9.1.0) as described in Methods.

Crystallography data analysis performed with XDS (VERSION Jan 31, 2020 BUILT=20200417), Coot (v. 0.9.5), ChimeraX (v. 1.1)/ISOLDE (v. 1.1), Refmac5 (v. 5.8.0267), and MOE (v. 2019.0102) 
Policy information about availability of data

All manuscripts must include a data availability statement. This statement should provide the following information, where applicable:

- Accession codes, unique identifiers, or web links for publicly available datasets

- A list of figures that have associated raw data

- A description of any restrictions on data availability

- All datasets generated and information presented in the study are available from the corresponding author on reasonable request.

- CryoEM structure data and model are available with accession code PDB 7RA8 (EMD-24347) and PDB 7RAL (EMD-24365)

- The X-ray structure data and model has been deposited with accession code PDB 7M7W for RBD-S2H97-S2X259.

- Interactive escape maps and structural visualizations can be found at: https://jbloomlab.github.io/SARS-CoV-2-RBD MAP Vir mAbs/.

- Raw Illumina sequencing data from deep mutational scanning experiments are available on NCBI SRA, BioSample SAMN18315604 (SARS-CoV-2 mutant selection data).

- Complete table of deep mutational scanning antibody escape fractions is provided on GitHub: https://github.com/jbloomlab/SARS-CoV-2-RBD_MAP_Vir_mAbs/ blob/main/results/supp_data/all_antibodies_raw_data.csv.

\section{Field-specific reporting}

Please select the one below that is the best fit for your research. If you are not sure, read the appropriate sections before making your selection. $\bigotimes$ Life sciences $\quad \square$ Behavioural \& social sciences $\square$ Ecological, evolutionary \& environmental sciences

\section{Life sciences study design}

All studies must disclose on these points even when the disclosure is negative.

Sample size For in vivo studies the sample size was chosen based on prior experience with this animal model.

Data exclusions Lack of detection of the human monoclonal antibody in the hamster sera at 48 post-administration (prophylactic setting) or at 4 days postinfection (therapeutic setting) was considered a criteria to identify animals that were not properly administered and to exclude them from the analysis

Replication Experimental assays were performed in biological duplicate or triplicate (or more) according to or exceeding standards in the field. We conducted all neutralization and antibody functional assays in biological duplicate, triplicate, or more, as indicated in relevant figure legends. In all cases, representative figure displays were appropriately replicated.

Randomization Hamsters were randomized to S2X259 and control treatment. For other experiments, randomization was not a relevant feature as we were applying a uniform set of techniques across a panel of candidate monoclonal antibodies

Blinding $\quad$ The hamster derived samples for RNA quantification and viral load titration were run by technicians who were blinded to treatment group of the analyzed samples

\section{Reporting for specific materials, systems and methods}

We require information from authors about some types of materials, experimental systems and methods used in many studies. Here, indicate whether each material, system or method listed is relevant to your study. If you are not sure if a list item applies to your research, read the appropriate section before selecting a response.

Materials \& experimental systems
$\mathrm{n} / \mathrm{a}$ Involved in the study
$\bigotimes$ Antibodies
$\bigotimes$ Eukaryotic cell lines
\ $\square$ Palaeontology and archaeology
$\square \bigotimes$ Animals and other organisms
$\square$ \uman research participants
邓 $\square$ Clinical data
$\bigotimes \mid \square$ Dual use research of concern

\begin{tabular}{l|l} 
Methods \\
\hline n/a & Involved in the study \\
$\square$ & $\square$ ChIP-seq \\
$\square$ & $\square$ Flow cytometry \\
$\square$ & $\square$ MRI-based neuroimaging
\end{tabular}



Bioscience 555779, anti-CD14 (BD Bioscience 562691), and anti-IgA (Southern Biotech 2050-09), and spike labeled with streptavidin Alexa-Fluor 647 (Life Technologies S21374)

* For FACS during deep mutational scanning, we used PE-conjugated goat anti-human-IgG (Jackson ImmunoResearch 109-115-098) and FITC-conjugated chicken anti-Myc (Immunology Consultants Lab, CYMC-45F).

* For VSV pseudotyped neutralization assays, media was supplemented with anti-VSV-G antibody (I1-mouse hybridoma supertant, ATCC CRL-2700)

* For SPR, immobilization was performed with anti-AviTag pAb (Genscript Cat \# A00674-40) and goat anti-human IgG Fc pAb, (Southern Biotech Cat \# 2014-01)

* For RBD ELISA, antibody binding was detected via goat anti-human IgG (Southern Biotech, 2040-04)

* For S1 shedding and binding FACS analysis, we used AF647-conjugated goat anti-human-IgG (Jackson ImmunoResearch 109-606-098)

* For antibody blockade of ACE2 binding, RBD was detected via goat anti-mouse IgG (Southern Biotech 1030-04)

* For detection of circulating mAb levels in hamster experiments, an anti-human LS mutation antibody was custom-made by BioRad AbD Serotec, and anti-human CH2 antibody from BioRad cat\# MCA5748G clone R10Z8E9

* Monoclonal antibodies (S309 and S2X259) were produced in-house using recombinant protein purification as described in the Methods. S2H97_GL and S2E12_GL were produced by ATUM.

All the commercial antibodies used in the study have been indicted with supplier name, catalog number. The monoclonal antibodies used as controls or for combination experiments were previously described by our group.

Validation

We discovered and describe S2X259 monoclonal antibody in this study. Target validation of this antibody was performed with multiple binding assays, in addition to cryoEM and $x$-ray crystallography structures were determined.

Reactivity of primary antibodies listed above is based on the information on manufacturer's homepages.

\section{Eukaryotic cell lines}

Policy information about cell lines

Cell line source(s)

Cell lines used in this study were obtained from ATCC (HEK293T, Vero and Vero-E6), ThermoFisher Scientific (Expi CHO cells, FreeStyle ${ }^{\mathrm{TM}} 293-\mathrm{F}$ cells and Expi293F $\mathrm{F}^{\mathrm{TM}}$ cells) or were generated via lentiviral trasduction (Expi CHO-S, HEK293T-ACE2).

Authentication

None of the cell lines used were authenticated

Mycoplasma contamination

Cell lines were tested for mycoplasma contamination

Commonly misidentified lines

(See ICLAC register)

No commonly misidentified cell lines were used in the study

\section{Animals and other organisms}

Policy information about studies involving animals; ARRIVE guidelines recommended for reporting animal research

Laboratory animals

Wild animals

Field-collected samples

Ethics oversight

Note that full information on the approval of the study protocol must also be provided in the manuscript. Janvier Laboratories and used in the study

The study did not involve wild animals

No field collected samples were used in the study
6-10 week-old female Syrian hamsters (Mesocricetus auratus) were Syrian hamsters (Mesocricetus auratus) were purchased from

This work was conducted in the high-containment A3 and BSL3+ facilities of the KU Leuven Rega Institute (3CAPS) under licenses AMV 30112018 SBB 21920180892 and AMV 23102017 SBB 21920170589 according to institutional guidelines. Housing conditions and experimental procedures were approved by the ethical committee of animal experimentation of KU Leuven (license P065-2020).

\section{Human research participants}

\section{Policy information about studies involving human research participants}

Population characteristics

Recruitment

Ethics oversight
Participants were identified by prior SARS-CoV-2 infection for PBMC isolation. Blood drawn from donor S2X (male, 52 yearsold) was obtained at day 75 after symptoms onset.

For recovered individual samples were collected between 14 and 75 days after symptoms onset, for vaccinees samples were collected between 13 and 20 days after the second dose of mRNA-based SARS-CoV-2 vaccine.

Patients were recruited on the basis of prior SARS-CoV-2 infection or vaccination in the hospital or outpatient setting. Patients were healthy volunteers who donated blood after being informed about the study. The only exclusion criteria used were HIV or other debilitating disease, but other information about diagnosis and treatment was not collected.

Study protocols for antibody isolation were approved by the local Institutional Review Board (Canton Ticino Ethics 


\section{Flow Cytometry}

\section{Plots}

Confirm that:

\The axis labels state the marker and fluorochrome used (e.g. CD4-FITC).

Х The axis scales are clearly visible. Include numbers along axes only for bottom left plot of group (a 'group' is an analysis of identical markers).

$\bigotimes$ All plots are contour plots with outliers or pseudocolor plots.

\A numerical value for number of cells or percentage (with statistics) is provided.

\section{Methodology}

Sample preparation

Instrument

Software

Cell population abundance

Gating strategy
Yeast were induced for cell-surface expression, incubated with the primary antibody from the panel, and secondary labeled with anti-Myc and anti-Fc fluorescent conjugates as described in the Methods.

FACSAria II and ZE5 BioRad

FACSDiva and Everest 3.0

We work with homogenous yeast libraries in our deep mutational scanning experiments, therefore cell population abundance is not a detail of our approach.

Yeast expressing unmutated SARS-CoV-2 are labeled at $1 x$ and $0.01 x$ the concentration used for library labeling. Gates are drawn on the basis of these controls to standardize selection stringency across expreriments. Gating schemes are shown in Extended Data Fig. 1c and 7a

$\bigotimes$ Tick this box to confirm that a figure exemplifying the gating strategy is provided in the Supplementary Information. 